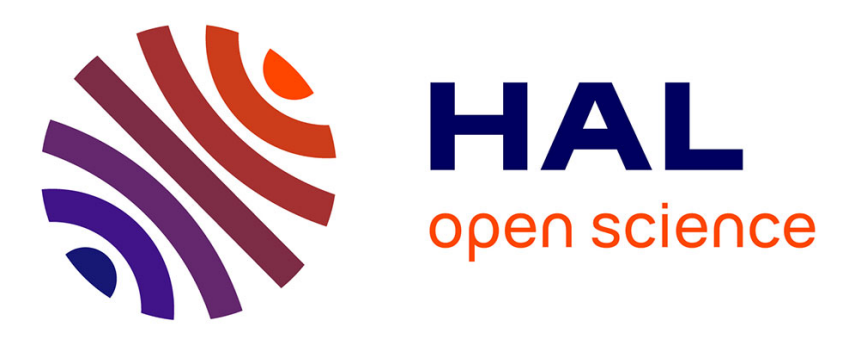

\title{
Forces on a boiling bubble in a developing boundary layer, in microgravity with g-jitter and in terrestrial conditions
}

Cees van Der Geld, Catherine Colin, Quint Segers, V.H. Pereira da Rosa, Harunori Yoshikawa

\section{To cite this version:}

Cees van Der Geld, Catherine Colin, Quint Segers, V.H. Pereira da Rosa, Harunori Yoshikawa. Forces on a boiling bubble in a developing boundary layer, in microgravity with g-jitter and in terrestrial conditions. Physics of Fluids, 2012, vol. 24, 10.1063/1.4743026 . hal-00873953

\section{HAL Id: hal-00873953 https://hal.science/hal-00873953}

Submitted on 16 Oct 2013

HAL is a multi-disciplinary open access archive for the deposit and dissemination of scientific research documents, whether they are published or not. The documents may come from teaching and research institutions in France or abroad, or from public or private research centers.
L'archive ouverte pluridisciplinaire HAL, est destinée au dépôt et à la diffusion de documents scientifiques de niveau recherche, publiés ou non, émanant des établissements d'enseignement et de recherche français ou étrangers, des laboratoires publics ou privés. 


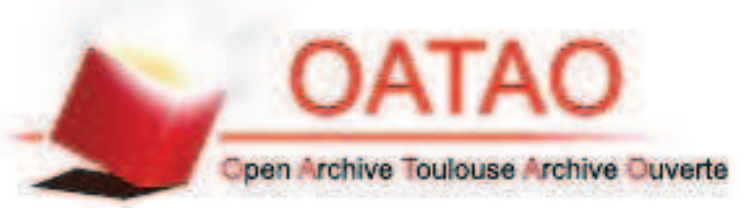

\section{Open Archive TOULOUSE Archive Ouverte (OATAO)}

OATAO is an open access repository that collects the work of Toulouse researchers and makes it freely available over the web where possible.

This is an author-deposited version published in : http://oatao.univ-toulouse.fr/ Eprints ID : 9790

To link to this article :DOI :10.1063/1.4743026

URL : http://dx.doi.org/10.1063/1.4743026

To cite this version Van der Geld, Cees and Colin, Catherine and Segers, Quint and Pereira da Rosa, V.H. and Yoshikawa, Harunori Forces on a boiling bubble in a developing boundary layer, in microgravity with g-jitter and in terrestrial conditions. (2012) Physics of Fluids, vol. 24 ( $\left.{ }^{\circ}{ }^{\circ} 082104\right)$. ISSN 1070-6631

Any correspondance concerning this service should be sent to the repository administrator: staff-oatao@,1istes-diff.inp-toulouse.fr 


\title{
Forces on a boiling bubble in a developing boundary layer, in microgravity with $g$-jitter and in terrestrial conditions
}

\author{
C. W. M. van der Geld, ${ }^{1}$,a) C. Colin, ${ }^{2}$ Q. I. E. Segers, ${ }^{1}$ \\ V. H. Pereira da Rosa, ${ }^{1}$ and H. N. Yoshikawa ${ }^{2}$ \\ ${ }^{1}$ Department of Mechanical Engineering, Eindhoven University of Technology, \\ P.O. Box 513, 5600 MB Eindhoven, The Netherlands \\ ${ }^{2}$ Inst. de Mécanique des Fluides de Toulouse, UMR CNRS/INPT/UPS 5502, \\ 2 Avenue Camille Soula, 31400 Toulouse, France
}

\begin{abstract}
Terrestrial and microgravity flow boiling experiments were carried out with the same test rig, comprising a locally heated artificial cavity in the center of a channel near the frontal edge of an intrusive glass bubble generator. Bubble shapes were in microgravity generally not far from those of truncated spheres, which permitted the computation of inertial lift and drag from potential flow theory for truncated spheres approximating the actual shape. For these bubbles, inertial lift is counteracted by drag and both forces are of the same order of magnitude as $g$-jitter. A generalization of the Laplace equation is found which applies to a deforming bubble attached to a plane wall and yields the pressure difference between the hydrostatic pressures in the bubble and at the wall, $\Delta p$. A fully independent way to determine the overpressure $\Delta p$ is given by a second Euler-Lagrange equation. Relative differences have been found to be about $5 \%$ for both terrestrial and microgravity bubbles. A way is found to determine the sum of the two counteracting major force contributions on a bubble in the direction normal to the wall from a single directly measurable quantity. Good agreement with expectation values for terrestrial bubbles was obtained with the difference in radii of curvature averaged over the liquid-vapor interface, $\left\langle\left(1 / R_{2}-1 / R_{1}\right)\right\rangle$, multiplied with the surface tension coefficient, $\sigma$. The new analysis methods of force components presented also permit the accounting for a surface tension gradient along the liquid-vapor interface. No such gradients were found for the present measurements.
\end{abstract}

\section{INTRODUCTION}

The general aim of much boiling research has been in the past, and still is today, the prediction of histories of bubble size and shape. ${ }^{1}$ In particular, the bubble volume at detachment plays a role in much mechanistic modeling of boiling and prediction of heat flux. ${ }^{2}$ Knowledge of the forces acting on a bubble is not only essential to predict its trajectory; it may serve as a basis to build correlations for heat transfer ${ }^{1}$ or mechanistic models ${ }^{3}$ upon. Force expressions are required to infer accelerations from Newton's second law of motion. The resulting second order differential equations for the position of the bubble are subsequently integrated and supplied with an appropriate set of initial positions and velocities in order to predict trajectories. Some of the forces involved result from the flow field of the fluid around the bubble. These so-called hydrodynamic forces can in principle be determined by solving local balances of mass and momentum in a numerical approach (CFD). The hydrodynamic forces are alternatively determined from empirical correlations which are usually expressions in terms of the local, instantaneous velocities of the bubble and the undisturbed flow field. ${ }^{4}$ Axisymmetric bubble growth in pool boiling at a horizontal surface in an otherwise quiescent

\footnotetext{
a) Author to whom correspondence should be addressed. Electronic mail: c.w.m.v.d.geld@tue.nl.
} 
liquid has successfully been predicted in the above Newtonian approach with analytical means with the neglect of inertia. ${ }^{5}$ In this case, possible bubble shapes depend on the ratio of capillary to gravity forces. Detachment occurs in axisymmetric pool boiling if no possible shapes for a given bubble volume and a given foot radius exist. ${ }^{5}$ Bubble detachment in pool boiling has also been investigated by direct numerical simulation. ${ }^{6}$

The present study is based on an alternative to the above-mentioned Newtonian treatment of bubble dynamics. It utilizes Euler-Lagrange equations which have proven to be a convenient approach if a bubble is deforming. ${ }^{7,4,8,9}$ This Euler-Lagrange approach facilitates extension of the above-mentioned Newtonian approach to bubbles without axisymmetry and to bubbles with an axisymmetric shape in a uniform flow field. In this paper, the approach is used to analyze new bubble growth measurements performed in flow boiling on earth and in parabolic flights, with the same set-up in identical process conditions. The bubbles thus measured will be named terrestrial and microgravity bubbles, respectively.

Ways will be explored to determine the sum of two major force contributions in the direction normal to the wall directly from a single measured quantity, rather than from assessments of these forces individually. Terrestrial bubbles with more or less known growth characteristics will be used to examine solutions presented. Particular attention will be given to the determination of the pressure inside the bubble, the so-called overpressure. Two independent ways to determine the overpressure will result from the Euler-Lagrange analysis in a natural way and results will be compared for both terrestrial and microgravity bubbles. In the literature, sometimes the Laplace equation is used to assess the overpressure. ${ }^{10,11}$ The present paper will present another expression, based on the first Euler-Lagrange equation for the isotropic component of the contour, which is valid for deformed bubbles. To satisfy a balance of estimated force components normal to the wall, a correction parameter is often used to multiply the overpressure with. ${ }^{12,13}$ The present analysis will be shown to be accurate enough not to need such a correction parameter and is useful for the development of point force models.

As for differences with our previous publications, the new analyzing method may appear to be a straightforward extension of the analysis of previous publications, but it is not. The bubbles studied in Ref. 8 were deforming in the proximity of a plane wall but were without a bubble foot at the wall. In Refs. 10 and 11, the bubble was attached to the wall, but it was assumed to have a spherical shape. The bubbles studied in Refs. 7 and 9 had exactly the shape of truncated spheres while those of the present study do not. In Ref. 7, only one governing equation was considered and in the present study more. In Refs. 7 and 9, the overpressure force exactly balances the capillary force, which means that the sum of these two dominant forces vanishes identically to zero. This is obviously impossible for terrestrial bubbles in the experiments of the present study and the new analysis is therefore for arbitrary bubble shape of a bubble with a foot at a plane wall. Buoyancy will be shown to be balanced by the sum of the two dominant forces and only slight deformation of the bubble is necessary to resettle this balance during bubble growth.

\section{EXPERIMENTAL}

The experimental test rig was developed at Institut de Mécanique des Fluides de Toulouse (IMFT) in France and the test section and bubble generator used for the experiments described in this paper at Eindhoven University of Technology (TU/e). The experiments were conducted by IMFT, with people of TU/e participating by invitation, during the 50th Parabolic Flight Campaign of May 2009 at Novespace, Bordeaux in France. The same setup was used for terrestrial experiments at IMFT. Microgravity during the elliptic top part of the airplane trajectory lasted for $21 \mathrm{sec}$.

The loop (Fig. 1) comprises a straight inlet section of $360 \mathrm{~mm}$ with a diverging and converging test section and a honeycomb in between. The inlet section is followed by a straight channel of $600 \mathrm{~mm}$ to make the flow fully developed in the test section $(170 \mathrm{~mm}$ length) positioned downstream. Internally, the rectangular channels measures $5 \times 40 \mathrm{~mm}^{2}$. A constant pressure volume compensator is used to keep the system pressure constant with the aid of an air volume behind a membrane. Fluid is HFE7000 that boils at $34^{\circ} \mathrm{C}$ at 1 bar. System pressure was set at 1.1 bar corresponding to 


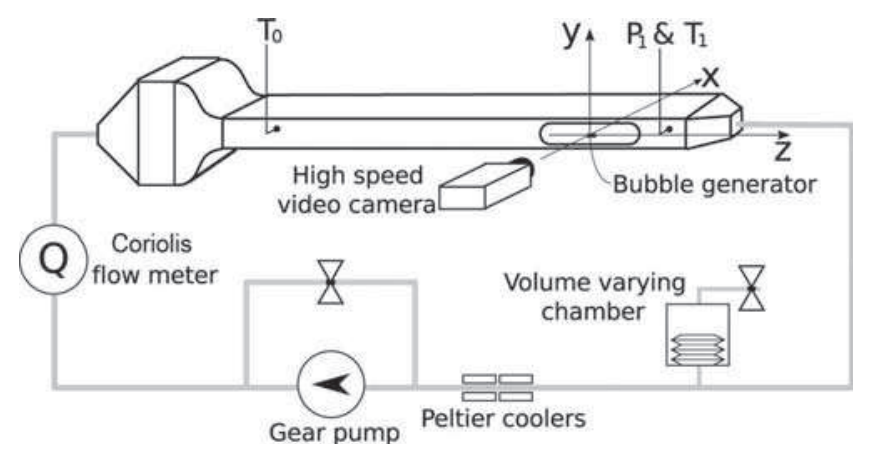

FIG. 1. Schematic of test rig.

a saturation temperature of $37^{\circ} \mathrm{C}$. Inlet subcooling was $4{ }^{\circ} \mathrm{C}$, so inlet temperature of the liquid was $33{ }^{\circ} \mathrm{C}$. Fluid was degassed for $5 \mathrm{~h}$ before having it filling the test loop which was made vacuum.

Test section is made of stainless steel with the aid of electric discharge machining. A glass bubble generator is positioned in the center of the test section such that an artificial cavity, with a volume of about $1 \mathrm{~mm}^{3}$, ends in an elliptical mouth in the very center of the channel (Fig. 2). The major axis of the ellipse was in flow direction and measured $0.17 \mathrm{~mm}$, while the shortest axis measured $0.033 \mathrm{~mm}$; area of the cavity mouth is $0.018 \mathrm{~mm}^{2}$. This cavity mouth is positioned at a distance of $0.493 \mathrm{~mm}$ of the sharp edge of the bubble generator (Fig. 2).

Thin films are sputtered with microwaves onto the glass to get an area of about $1 \times 1 \mathrm{~mm}^{2}(0.947$ $\times 0.789 \mathrm{~mm}^{2}$ to be precise) around the cavity mouth covered by a titanium coating, with a typical thickness of $500 \mathrm{~nm}$. Bubble radius is less than $1 \mathrm{~mm}$ so heat flux from the thin film to the bubble is not hampered by size limitations of the heating area. The titanium coating has a good adherence to the glass. The two leads towards the heated bubble generation site are made of titanium with a gold layer on top, see Fig. 2 where the gold is shown as a lighter grey on line. Electric resistance is only high at the heating area of $1 \times 1 \mathrm{~mm}^{2}$. The leads are connected to a power supply.

The flow approaching the sharp edge of the glass bubble generator is as uniform as it can be in the channel used. Downstream of the sharp edge a boundary layer starts developing. Two

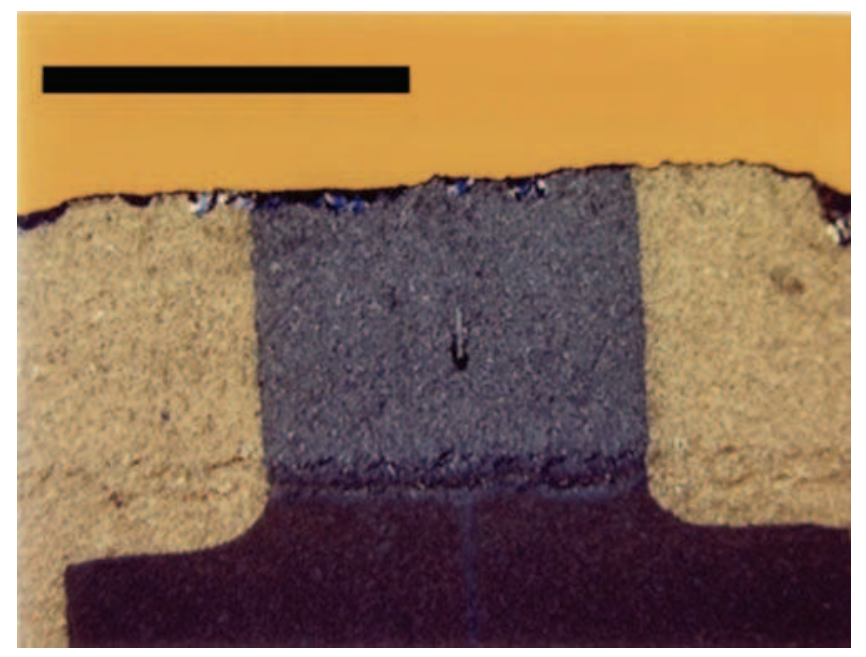

FIG. 2. View from above onto the flat surface on which bubbles are created. The irregular rim in the top of this picture is the edge of the coated glass bubble generator and below this edge the bubble generator is seen, with uncoated glass in black and with golden leads (shown lighter grey on line) coming from left and right towards the dark square around the artificial cavity. Heat is generated uniformly in the coating of the latter square (darker grey, bluish on line). The black bar on the top left of the figure is a scale that measures $1 \mathrm{~mm}$. Flow direction is from top to bottom, normal to the frontal edge. The artificial cavity ends as an ellipsoidal hole with axis $0.17 \mathrm{~mm}$ and $0.033 \mathrm{~mm}$. 


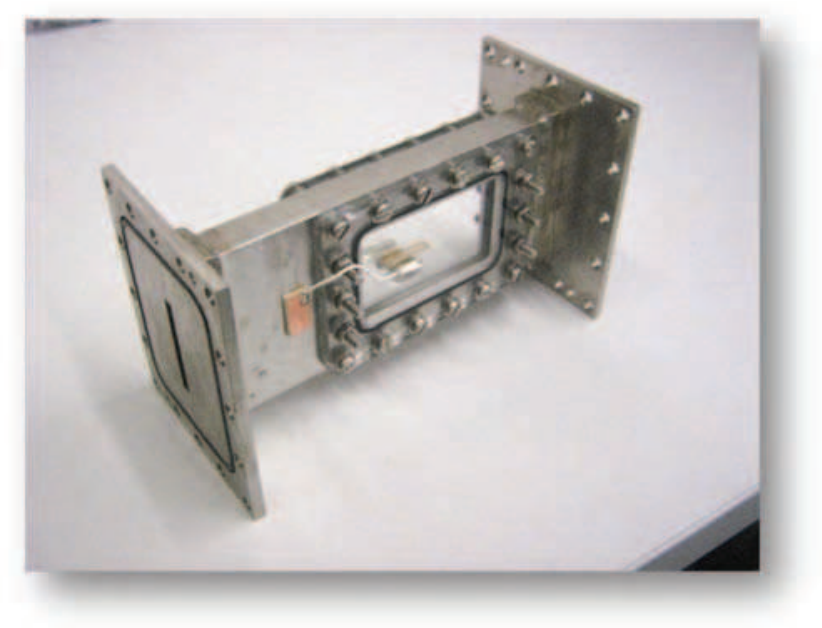

FIG. 3. Photograph of the bubble generator mounted in the stainless steel test section. The slit on the left is the outlet and measures $40 \times 5 \mathrm{~mm}^{2}$.

polycarbonate (Lexan ${ }^{\mathrm{TM}}$, fabricated by SABIC Innovative Plastics, The Netherlands) windows permit visual observations from the side (Fig. 3). Estimates, to be discussed in Sec. IV A, show that while bubble nucleation occurs inside the boundary layer, most of the liquid-vapor interface is outside it during bubble growth until the bubble detaches and moves downstream, see Fig. 4.

Bulk liquid flow rate is measured with a Coriolis flow meter, type Micromotion R025S (Fig. 1). The measurements presented in this paper were performed with a liquid velocity that amounted to $0.0120 \pm 0.0004 \mathrm{~m} / \mathrm{s}$ at the location of the bubble generator, with a profile that fairly constant at the scale of bubble diameters measured. This profile was characterized by a series of PIV measurements. The flow can be laminar or turbulent depending of the bulk Reynolds number. For the results presented hereafter, it is a laminar fluid flow, with a bulk Reynolds number of about 300 based on a kinematic viscosity of $3.2 \times 10^{-7} \mathrm{~m}^{2} / \mathrm{s}$, a volume flow rate of $2.14 \times 10^{-6} \mathrm{~m}^{3} / \mathrm{s}$, channel widths 5 and $40 \mathrm{~mm}$. Accelerometers, to measure $g$-jitter (typically $0.045 \mathrm{~m} / \mathrm{s}^{2}$ ), temperature and pressure sensors were recorded at $2 \mathrm{kHz}$ and video-recordings of bubble growth were made at $500 \mathrm{~Hz}$. Diffuse background illumination with a halogen lamp was applied and each image was after cropping, to save storage capacity, composed of $1280 \times 700$ pixels. Size calibration was done both with a scale with equidistant lines of $0.1 \mathrm{~mm}$ and by moving the camera with the aid of a micrometer

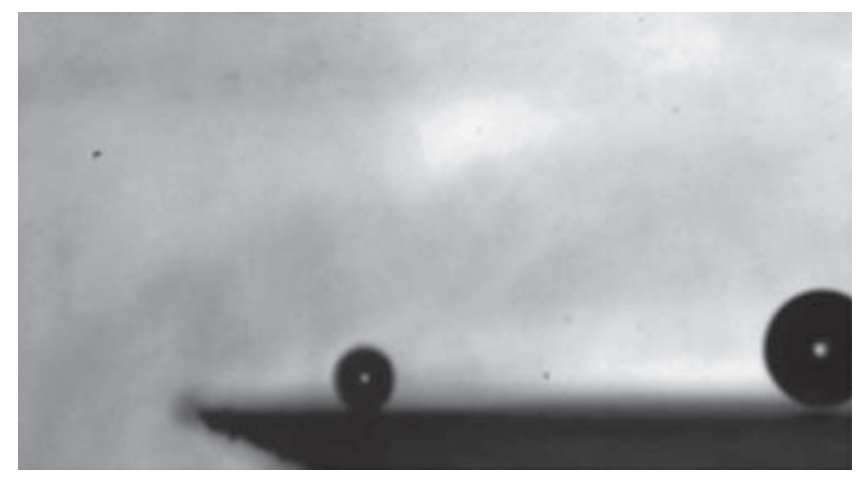

FIG. 4. Bubble growing in a boundary layer in microgravity; approaching liquid velocity $V=1.2 \mathrm{~cm} / \mathrm{s}$, coming from the left. Diameter of the bubble on the LHS is $0.2 \mathrm{~mm}$. Asymmetry between front and rear of this small bubble is barely observed. The large bubble on the RHS is fully detached from the wall directly after its creation by coalescence of two smaller bubbles. In the corresponding movie, the bubble is observed to grow and slide downstream, to the RHS of the figure. 
screw gauge. Conversion factor is $0.00198 \mathrm{~mm} /$ pixel with an uncertainty of $2 \%$. Each photograph of a video-recording is separately analyzed, in a way described in Sec. III A.

\section{FORCE ANALYSES}

\section{A. Determination of generalized coordinates}

Each 2D-image of a bubble is in grayscale and inverted in grayscale to facilitate the subtraction of a reference figure without a bubble but with the physical wall. The latter reference figure not always exists if the solid wall is moving in the camera view (microgravity experiments). The resulting image is cropped and rotated before a median filter is applied once and a Gaussian filter applied three times in order to remove inaccuracies due to pixel resolution limitations. Then the zero-cross method is used to determine points on the liquid-gas interface. These points are subsequently connected in an edge-line determination procedure.

Since the fluid flow parallel to the wall might have caused asymmetry in the projected shape of the bubble, the smoothed bubble contour is split into two halves in the following way. First, a chosen wall is selected parallel to the actual, solid wall on which the bubble is attached, see Fig. 5. The chosen wall could be on top of the actual wall but is usually selected to be positioned in the fluid region at a small distance from the actual wall. This is possible since the force balances that will be derived need to be satisfied for arbitrary bubble volume bounded by part of the measured contour and a chosen wall. Only the estimates of the hydrodynamic forces to be presented decrease in accuracy with increasing distance of the chosen wall to the actual wall. This, however, will be seen to have hardly any consequence for the conclusions that will be drawn. The cross-section of the bubble contour with the chosen wall is a line. Let the length of this line be $2 r_{\text {foot }}$, which defines $r_{\text {foot }}$. Next, the top of the bubble is determined as the point furthest away from the chosen wall. Suppose that $\hat{y}$ is a unit vector of a Cartesian coordinate system in the projection plane pointing towards the chosen wall and normal to this wall. If the chosen wall has coordinate $y_{\text {wall }}$ and the top of the bubble has coordinate $y_{t o p}$ then the maximum height of the bubble is given by $y_{\text {wall }}-y_{\text {top }}$. The line through the top of the bubble normal to the actual wall is the line on which the center of the bubble, $\mathrm{O}$, will be found and is also the line that splits the bubble in two halves. The right hand side of the bubble by definition is the half which is downstream of the fluid flow. This right hand side is mirrored to the line through the top and $\mathrm{O}$ in order to obtain a fully symmetrical bubble contour. The bubble is assumed

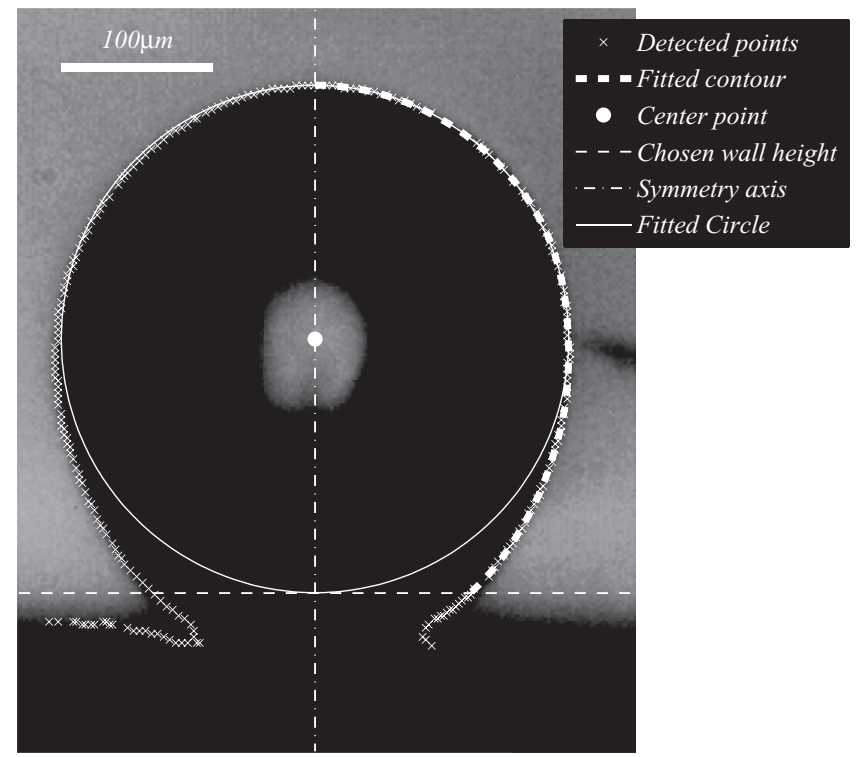

FIG. 5. Measured contour points, fitted contour and chosen wall. 


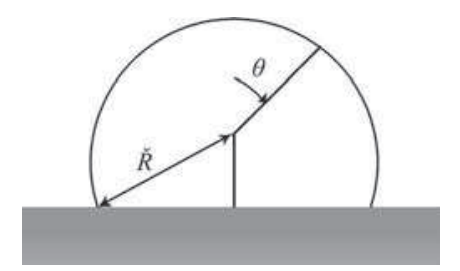

FIG. 6. Polar coordinate system with radial distance of the center to the bubble foot, $\check{R}$.

to be axisymmetric around the line through the top and $\mathrm{O}$ and the applicability of this assumption is assessed in the following way. The cross-section of the present contour with the chosen wall has a length, which is compared with $2 r_{\text {foot }}$. Differences turn out to be about $2 \%$, which is considered as acceptable in view of the difficulty to measure the bubble foot and the similarity to a sphere of the top of the bubble (Fig. 5). This similarity is quantified in the next paragraph. The length $r_{f o o t}$ is retained in the following in order to increase accuracy of the determination of the area of the bubble in contact with the chosen wall.

The center of the bubble is determined as follows. A circle with radius $R_{\text {sphere }}$ is fitted through contour points near the top of the bubble. When gravity is terrestrial, the shape of the bubble is such that the radius of curvature of the bubble is continuously varying near the top and depends on coordinate $y$. Because of this there is some arbitrariness in the determination of $R_{\text {sphere }}$. However, the present analysis is aimed at a comparison of measured contours with those of truncated spheres and a value of a circle radius is therefore needed which makes the arbitrariness unavoidable. It is obvious that the fewer contour points are used to determine $R_{\text {sphere }}$, the closer its value will be to one of the radii of curvature at the bubble top and the more unique the value of $R_{\text {sphere }}$. For comparison with the truncated sphere shape it is better to select more points at the contour. The determination of $R_{\text {sphere }}$ is done by least square fitting of a circle through the selected contour points. Figure 5 shows a typical result.

In the analysis of histories of boiling bubbles shaped as truncated spheres it is customary to use $\left(h, R_{\text {sphere }}\right)$ as the coordinates of the bubble. For this reason, distance $h$ of the center $\mathrm{O}$ from the chosen wall is considered a generalized coordinate in the present analysis. Inertia and drag forces will be estimated using only $h$ and $R_{\text {sphere }}$ as coordinates, but only because no expressions are available for inertia and drag that take further deformation into account. If gravity is terrestrial and/or governing Reynolds numbers are big, however, deformation cannot be neglected. In our experiments at microgravity, typical value of the Weber number, We $=\rho_{L} V^{2} 2 R_{\text {sphere }} / \sigma$, is 0.002 $\left(V=0.012 \mathrm{~m} / \mathrm{s}, R_{\text {sphere }}=0.15 \mathrm{~mm}, \rho_{L}\right.$ is mass density of the liquid, and $\sigma$ is surface tension coefficient), which proves that the bubble deformation is negligible in this case. Governing Reynolds numbers are based on length scale $R_{\text {sphere }}$ and velocity scales $\dot{R}_{\text {sphere }}=d R_{\text {sphere }} / d t$ or $U$, the time rate of change of $h$, or $V$, the approach velocity parallel to the wall of the fluid at the center $\mathrm{O}$, which is taken to be a uniform approach velocity; $t$ denotes time. Since deformation cannot always be neglected, Legendre coefficients are determined as generalized coordinates further to $h$ as follows

Define $x=\cos (\theta)$ with $\theta$ the polar angle measured from the top of the bubble in the polar coordinate system centered at center $\mathrm{O}$, see Fig. 6 . Let $\theta_{1}$ be the angle corresponding to the foot of the bubble at the chosen wall, corresponding to radial distance $\check{R}$ of this foot, such that $\cos \left(\theta_{1}\right)$ $=-h / \not ̌ R$. Only if for all $\theta$ radial distance $R(\cos (\theta))$ of the contour equals $R_{\text {sphere }}$ the shape is that of a truncated sphere with radius $\check{R}=R_{\text {sphere }}$ and height $h$ above the chosen wall.

Let the holonomic constraint $r=R(\cos (\theta), t)$ represent the measured contour on $\left[\cos \left(\theta_{1}\right), 1\right]$. This constraint is applicable to all measured shapes. The extension $R$ of $R$ is defined by

$$
\begin{gathered}
R_{3}(x)=R(x) \text { if } x \geq \cos \left(\theta_{1}\right) \text { and if } x<\cos \left(\theta_{1}\right) \text { then } \\
R_{3}(x)=\check{R}+d_{1}\left(x-\cos \left(\theta_{1}\right)\right)+d_{2}\left(x-\cos \left(\theta_{1}\right)\right)^{2}
\end{gathered}
$$




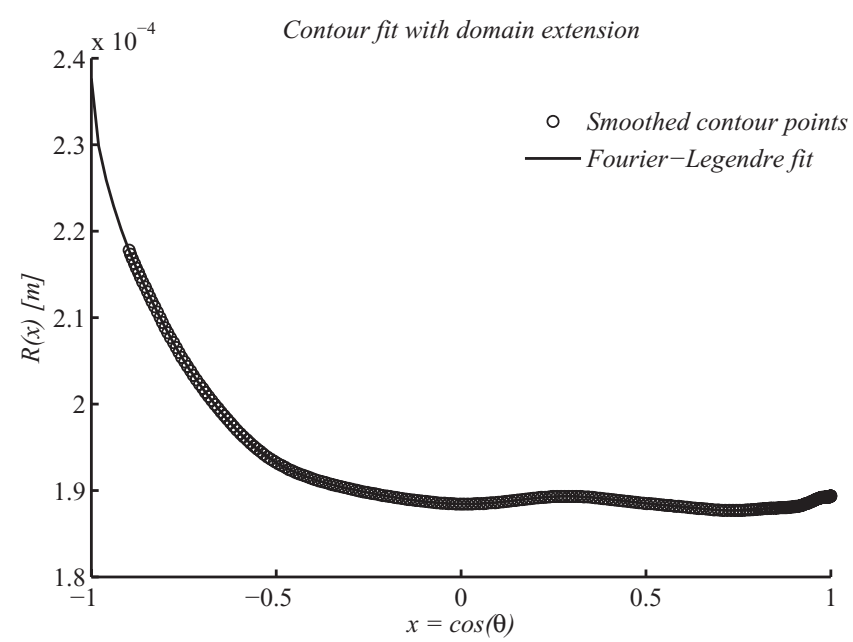

FIG. 7. Typical example of measured contour points ("Data points") with second order extension to improve accuracy of curvature determination at the bubble foot $(x=-0.85)$ and Legendre fit (solid line).

with constants $d_{1}$ and $d_{2}$ such that the curvature at $\cos \left(\theta_{1}\right)$ can accurately be evaluated. This requires a smooth, twice differentiable continuation at $\cos \left(\theta_{1}\right)$. Let $L e_{j-1}$ denote the Legendre polynomial of order $j-1\left(L e_{0}=1\right)$. Fourier-Legendre coefficients of $R, b_{j}$, are defined by

$$
R_{s}=\Sigma_{j} \tilde{b}_{j} L e_{j-1}(\cos (\theta))=\Sigma_{j} \tilde{b}_{j} L e_{j-1}(x),
$$

and the $\tilde{b}_{j}$ are determined by integration in the usual way. Indices in the summation run from 1 to 25. For sake of brevity, ю is defined by

$$
Ю=\cos \left(\theta_{1}\right) .
$$

Let

$$
a_{1}=\partial R /\left.\partial x\right|_{ю} \text { and } a_{2}=1 / 2 / 2 \partial^{2} R /\left.\partial x^{2}\right|_{ю} .
$$

The smooth continuation obviously requires that $d_{1}=a_{1}$ and $d_{2}=a_{2}$. Figure 7 shows a typical example of an extended bubble contour $R(x)$.

It turns out with properly selected distance $h, \tilde{b}_{2}$ is much less than both $\tilde{b}_{1}$ and $h$. The absolute value of the ratio of $\frac{\partial \tilde{b}_{2}}{\partial t}$ to $\frac{\partial h}{\partial t}=\dot{h}=U$ is less than $2 \%$ in all measurements. Since for a free bubble $h$ and $\tilde{b}_{2}$ are dependent, the following parameters are selected as generalized coordinates: $b_{1}=\tilde{b}_{1}$, $b_{2}=h, b_{3}=\tilde{b}_{3}, b_{4}=\tilde{b}_{4}, b_{5}=\tilde{b}_{5}$, etc. The value of $b_{1}$, or $\tilde{b}_{1}$, can be seen as the mean distance of the bubble contour to the center point. The value of $b_{1}$ is close to that of $R_{\text {sphere }}$ if the bubble is nearly a truncated sphere. In integrals and other expressions involving contour fit $R(x)$ or $R(x)$, all $\tilde{b}_{j}$-coefficients are taken into account. In one case this yields a correction coefficient $c_{1}=1+\dot{\tilde{b}}_{2} / U$ in an integral.

Two brackets will be used to indicate an average over the entire gas-liquid interface. For example, the average $\langle R\rangle$ is given by

$$
\langle R\rangle=\int_{\text {ю }}^{1} d x R(x) /(1-ю)=\left\{\int_{0}^{\theta_{1}} d \theta R(\theta) \sin (\theta)\right\} /\left(1-\cos \left(\theta_{1}\right)\right) .
$$

\section{B. Determination of the main force components}

When the generalized coordinates defined in Sec. III A are used, Newton's law of motion has to be transformed and expressions for the corresponding generalized forces have to be derived. This has been done in a derivation that starts from the so-called mechanical energy balance for the fluid surrounding the bubble. ${ }^{9}$ The resulting time rate of change of the kinetic energy, $T$, can be given the 
form

$$
\frac{d T}{d t}=\Delta p d V_{b} / d t+\sigma d A_{b} / d t+\ldots,
$$

where the dots indicate expressions whose components are specified below. Volume and area of the bubble are $V_{b}$ and $A_{b}$, respectively, while $\sigma$ is the surface tension coefficient and $\Delta p$ the pressure difference between the homogeneous pressure inside the bubble and the pressure in the liquid near the solid wall on which the bubble is footed. There is no restriction on kinetic energy $T$ in this stage; it accounts for turbulence as well as for creeping flow. The RHS of this equation can be written as the sum of products of generalized forces, $F_{j}$, and generalized velocities, $q_{j}=\dot{b}_{j}: d T / d t=\sum_{\mathrm{j}} F_{j}$ $q_{j}$. It is now assumed that the fluid flow is solenoidal which is, for example, satisfied if the fluid is incompressible. The LHS of the above equation can then be written as the sum of the time rates of change of a purely irrotational component, $T^{\text {irrot }}$, of a purely vector potential component, $T^{\text {vort }}$, and of a mixed component, $T^{\text {mixed }}$. This is because the velocity field is the sum of an irrotational scalar component and a solenoidal vector potential component. ${ }^{14}$ The component $T^{\text {irrot }}$ can be expressed in terms of added mass coefficients. In the general case, when the vorticity contribution cannot be neglected, equations of the following form are derived ( $j$ runs from 1 to 25 ):

$$
-F_{j}^{\text {inertia }}-F_{j}^{\text {vort }}=F_{j}^{\Delta p}+F_{j}^{\sigma}+F_{j}^{g}+F_{j}^{\text {drag }},
$$

where force $F_{j}{ }^{\text {vort }}$ accounts for the purely vector potential and mixed components. On the RHS of Eq. (2), each $F_{j}$ represents a generalized force which will be defined below and which corresponds to generalized coordinate $b_{j}$. It is noted that the forces which are here denoted as inertia forces, $F_{j}^{\text {inertia }}$, stem from $T^{\text {irrot }}$ and retain their form whatever the values of $T^{v o r t}$ and $T^{\text {mixed }}$. The reason for this is that the added mass forces and added mass coefficients stay the same in viscous flows. ${ }^{4,15} \mathrm{~A}$ uniform approaching flow with velocity $V$ parallel to the wall yields a potential flow lift contribution to the force balance in the direction perpendicular to the solid wall (labeled with "2"), which is in $F_{2}{ }^{\text {inertia }}$. Lift contributions to this force balance, which are related to vorticity are accounted for by $F_{2}{ }^{\text {vort }}$. Lift and other hydrodynamic forces are further discussed below.

The gravitational forces, $F_{j}{ }^{g}$, as well as the surface tension forces, $F_{j}{ }^{\sigma}$, turn out to be expressible as integrals over the gas-liquid interface. The surface integrals mostly can be expressed in quantities prevailing at the bubble foot, the part of the bubble in contact with the solid wall. These quantities are mostly familiar ones. However, the measurement accuracy of these quantities is usually not high because of the so-called mirage effect ${ }^{16}$ and since the wall is not measured as a sharp line (Fig. 5). This is important for the analysis of forces based on measured shapes of a bubble attached to a wall. The present study will compare quantities measured at the bubble foot with values determined from corresponding surface integrals.

Equation (2) is valid for $j=1$ to 25 , which implies that 25 governing equations exist. The present analysis focuses on the isotropic component $b_{1}$ and translational motion described by $b_{2}=h$ because of the wish to compare with truncated sphere bubbles described by the two length scales $R_{\text {sphere }}$ and $h$. This means that the system of equations given by (2) is reduced to two equations in 25 generalized coordinates and 25 generalized velocities which can be given the following form:

$$
\begin{aligned}
& -F_{1}^{\text {inertia }}\left(b_{j}, \dot{b}_{j}, \ddot{b}_{j}\right)=F_{1}^{\text {vort }}\left(b_{j}, \dot{b}_{j}\right)+F_{1}^{\Delta p}\left(b_{j}\right)+F_{1}^{\sigma}\left(b_{j}\right)+F_{1}^{g}\left(b_{j}\right)+F_{1}^{\text {drag }}\left(b_{j}, \dot{b}_{j}\right), \\
& -F_{2}^{\text {inertia }}\left(b_{j}, \dot{b}_{j}, \ddot{b}_{j}\right)=F_{2}^{\text {vort }}\left(b_{j}, \dot{b}_{j}\right)+F_{2}^{\Delta p}\left(b_{j}\right)+F_{2}^{\sigma}\left(b_{j}\right)+F_{2}^{g}\left(b_{j}\right)+F_{2}^{\text {drag }}\left(b_{j}, \dot{b}_{j}\right) .
\end{aligned}
$$

Here, the occurrence of $\left(b_{j}, \dot{b}_{j}, \ddot{b}_{j}\right)$ indicates dependency on all generalized coordinates, generalized velocities, and generalized accelerations; $\ddot{h}$ denotes the second order derivative of $h$ with respect to time. As the inertia force will be seen to be linear in the generalized velocities, the above are two coupled second order differential equations in the generalized coordinates. Despite the fact that $F_{j}{ }^{\Delta p}, F_{j}{ }^{\sigma}$, and $F_{j}{ }^{g}(j=1,2)$ are only dependent on the generalized coordinates, i.e., on the shape of the bubble, they are the dominant forces that must be determined from experimental observations with a high accuracy. This determination is the subject of the remainder of this section. 
The first force on the RHS of (2) is $F^{\Delta p}$, the force due to the overpressure inside the bubble. The overpressure is defined by $\Delta p=\left(p_{b}-p_{w}\right), p_{b}$ being the homogeneous pressure inside the bubble and $p_{w}$ being the hydrostatic pressure in the fluid at the actual, solid plane wall. The overpressure force components, $F_{j}{ }^{\Delta p}$, follow from

$$
F_{j}^{\Delta p}=\Delta p \partial V_{b} / \partial b_{j},
$$

where $V_{b}$ denotes the volume of the bubble. In expression (3) the overpressure $\Delta p$ is an unknown which occurs in each governing equation, labeled by $j$, of (2). The overpressure inside the bubble is related to heat transfer and since the energy equation is not solved for the experimental conditions at hand it is a time-dependent quantity, which must be determined from measurement data. Hydrodynamic stresses near a bubble can be estimated as $1 / 2 \rho_{L} \dot{R}_{\text {sphere }}^{2}$ which is typically about $0.07 \mathrm{~Pa}$ for a microgravity bubble and $0.002 \mathrm{~Pa}$ for a terrestrial bubble. If hydrodynamic stresses near the bubble foot at a chosen wall (see Sec. II) can be neglected, the so-called dynamic stress boundary condition across the liquid-gas interface at the bubble foot gives the following expression for the overpressure $\Delta p$ :

$$
\Delta p=-\left.2 \sigma H\right|_{\text {foot }}-\rho_{L} g h_{\text {wall }},
$$

where $\left.H\right|_{\text {foot }}$ is the negative mean curvature at the bubble foot and $h_{\text {wall }}$ is the height of the chosen wall above the actual wall. An expression of the mean radius of curvature will be given below. A typical value of $-\left.2 \sigma H\right|_{\text {foot }}$ is $200 \mathrm{~Pa}$ and of $\rho_{L} g h_{\text {wall }}$ is $0.1 \mathrm{~Pa}$, which shows that the latter, hydrostatic term is only a minor correction. Moreover, the above estimates of hydrodynamic stresses are even less, and therefore negligible indeed. In the present study, the overpressure will be determined in various ways in an attempt to increase the accuracy of the measurement result. Ways other than with (4) to determine the overpressure are described below.

The partial derivatives $\partial V_{b} / \partial b_{j}$ in (3) are for $j=1$ and $j=2$ readily determined from the measured shapes with the aid of the following measurable quantities:

$$
\begin{gathered}
\left\langle R^{2}\right\rangle=\int_{ю}^{1} d x R^{2}(x) /(1-ю)=\left\{\int_{0}^{\theta 1} d \theta R^{2}(\theta) \sin (\theta)\right\} /\left(1-\cos \left(\theta_{1}\right)\right), \\
\left\langle R^{3}\right\rangle=\int_{ю}^{1} d x R^{3}(x) /(1-ю) .
\end{gathered}
$$

Note that ю $=\cos \left(\theta_{1}\right)=-h / \check{R}$. The first quantity, $\left\langle R^{2}\right\rangle$, is used to assess $\partial V_{b} / \partial b_{1}$ from

$$
\partial V_{b} / \partial b_{1}=2 \pi(1-ю)\left\langle R^{2}\right\rangle .
$$

For a truncated sphere, $\partial V_{b} / \partial b_{1}=2 \pi \check{R}(\check{R}+h)$, with $\check{R}=R_{\text {shpere }}$, since then $\left\langle R^{2}\right\rangle=\check{R}^{2}$. The volume of the bubble follows from

$$
V_{b}=2 / 3 \pi(1-ю)\left\langle R^{3}\right\rangle+1 / 3 \pi h\left(\check{R}^{2}-h^{2}\right) .
$$

It can be shown that

$$
F_{2}^{\mathrm{g}}=g \rho L V_{b}
$$

with $\rho_{L}$ being the mass density of the liquid. The other partial derivative of the bubble volume, for $j=2$ in (3), is given by

$$
\partial V_{b} / \partial b_{2}=\partial V_{b} / \partial h=1 / 3 \pi \check{R}^{2}-\pi h^{2}=\pi r_{\text {foot }}^{2}
$$

with $r_{\text {foot }}$, in principle equal to $\check{R} \sin \left(\theta_{1}\right)$, evaluated as half the width of the chosen wall line crossing the bubble contour (Sec. III A). 
The following definition is convenient in the force analysis below and represents the average of twice the mean curvature, $2 \mathrm{H}$, over the entire gas-liquid interface:

$$
\langle 2 H\rangle=\int_{0}^{\theta_{1}} d \theta R^{2}(\theta) \sin (\theta) 2 H / \int_{0}^{\theta_{1}} d \theta R^{2}(\theta) \sin (\theta),
$$

where $\int_{0}^{\theta_{1}} d \theta R^{2}(\theta) \sin (\theta)$ is equal to ${ }_{\text {ю }} \int^{1} d x R^{2}(x)=(1-ю)\left\langle R^{2}\right\rangle$. The mean radius of curvature is denoted with $H$ and is given ${ }^{17}$ as

$$
((1))^{-1 / 2}\left[1 / 2\left(R^{\prime} / R\right) \operatorname{ctg}(\theta)-1+1 / 2\left(R R^{\prime \prime}-R^{\prime 2}\right) /((1))\right],
$$

where $((1))=R^{2}+R^{\prime 2}$ with $R^{\prime}=\partial R / \partial \theta$, by definition and $R^{\prime \prime}$ by definition the second order derivative of $R$ with respect to $\theta$. For a truncated sphere, $H=-1 / \check{R}=-1 / R_{\text {shpere }}$. The generalized force $F_{2}^{\sigma}$ corresponding to $h$ is given by

$$
F_{2}^{\sigma}=2 \pi \sigma \int_{ю}^{1} d x 2 H R\left(c_{1} x R+\sqrt{\left(1-x^{2}\right)} R^{\prime}\right)
$$

with $c_{1}=\left(1+\dot{\tilde{b}}_{2} / U\right)$ a correction to velocity $U$ for the bubble motion as a whole; note that on the relevant domain of the polar angle, $\sin (\theta)=\sqrt{ }\left(1-x^{2}\right)$. The ratio of time derivative $\dot{\tilde{b}}_{2}$ to $\mathrm{U}$ is usually less than 0.02 for terrestrial bubbles and about -0.02 for the microgravity bubbles we measured. These values make $c_{1}$ effectively equal to 1 . Taking $c_{1}=1$, the force $F_{2}^{\sigma}$ is given by

$$
\begin{aligned}
& 2 \pi \sigma \int_{\text {ю }}^{1} d x((1))^{-1 / 2} \\
& \quad \times\left[x\left\{R^{\prime 2}-2 R^{2}+R((0)) /((1))\right\}+\left\{R^{\prime}((0)) /((1))-2 R R^{\prime}\right\} \sqrt{\left(1-x^{2}\right)}+R R^{\prime} x^{2} / \sqrt{\left(1-x^{2}\right)}\right]
\end{aligned}
$$

with $((0))=\left(R^{2} R^{\prime \prime}-R R^{\prime 2}\right)$. It can be proven that this expression reduces to

$$
-2 \pi r_{f o o t} \sigma \sin \left(\theta_{c}\right)
$$

with $\theta_{c}$ the contact angle at the bubble foot measured in the liquid. The minus sign indicates action into the direction opposite to $h$, so a force attracting the bubble towards the wall.

The generalized force $F^{\sigma}{ }_{1}$ corresponding to the isotropic component $b_{1}$ is given by

$$
F_{1}^{\sigma}=2 \pi \sigma \quad \int_{\text {ю }}^{1} d x((1))^{-1 / 2}\left[R R^{\prime} x / \sqrt{\left(1-x^{2}\right)}-2 R^{2}+R((0)) /((1))\right] .
$$

It can be proven that this expression reduces to

$$
\sigma\langle 2 H\rangle \partial V_{b} / \partial b_{1} \text {. }
$$

This expression reduces to $-4 \pi \sigma(\check{R}+h)$ for the case of a truncated sphere, with $\check{R}=R_{\text {shpere }}$, since $\langle 2 H\rangle=-2 / \check{R}$ then.

The definition

$$
\left\langle x R^{3}\right\rangle=\int_{ю}^{1} d x \times R^{3}(x) /(1-ю)
$$

makes it possible to express the gravity force $F_{1} \mathrm{~g}$ as follows:

$$
F_{1}^{\mathrm{g}}=g \rho_{L} 2 \pi\left(1-\text { ю) }\left(h\left\langle R^{2}\right\rangle+\left\langle x R^{3}\right\rangle\right) .\right.
$$

For a truncated sphere, (15) yields $g \rho_{L} \pi \check{R}(h+\check{R})^{2}$, with $\check{R}=R_{\text {shpere }}$.

Collecting (3) and (13) into (2), we obtain for the isotropic component the following equation (16) that can be considered as an extended Rayleigh-Plesset equation for a bubble attached to a plane wall. Expression (6) can be used to assess $\partial V_{b} / \partial b_{1}$ and (12) for $F_{1}{ }^{g}$,

$$
-F_{1}^{\text {inertia }}-F_{1}^{\text {vort }}=\Delta p \partial V_{b} / \partial b_{1}+\sigma\langle 2 H\rangle \partial V_{b} / \partial b_{1}+F_{1}^{g}+F_{1}^{\text {drag }} .
$$


The drag force $F_{1}{ }^{d r a g}$ will be specified in Sec. III C below. A good approximation for the overpressure is seen to be given by $-\sigma\langle 2 H\rangle$, since other contributions in (16) are usually less than this capillary term. This approximation is $2 \sigma / \check{R}$ in the case of a truncated sphere. A more accurate way to assess the overpressure $\Delta p$ is easily obtained by rewriting (16),

$$
\Delta p=-\sigma\langle 2 H\rangle-\left(F_{1}^{\text {inertia }}+F_{1}^{g}+F_{1}^{\text {drag }}\right) /\left(\partial V_{b} / \partial b_{1}\right)
$$

The difference $\left(-\left.2 \sigma H\right|_{\text {foot }}+\sigma\langle 2 H\rangle\right)$ is given by the $\left(F_{1}{ }^{\text {inertia }}+F_{1}{ }^{g}+F_{1}{ }^{\text {drag }}\right)$-term on the RHS of this equation.

Similarly, Eq. (17) for the second generalized coordinate, the distance of the center, $h$, to the wall, is obtained from (8),

$$
-F_{2}^{\text {inertia }}-F_{2}^{\text {vort }}=\Delta p \pi r_{\text {foot }}^{2}-2 \pi r_{\text {foot }} \sigma \sin \left(\theta_{c}\right)+g \rho_{L} V_{b}+F_{2}^{\text {drag }} .
$$

Capillary force $F_{2}^{\sigma}$ is for (17) determined with (11), but Eq. (10) yields more accurate values, usually. It can be proven that at the chosen wall

$$
\left.2 H\right|_{\text {foot }}=1 / R_{1}-\sin \left(\theta_{c}\right) / r_{\text {foot }},
$$

with $R_{1}$ a radius of curvature at the bubble foot while $1 / R_{2}=-\sin \left(\theta_{c}\right) / r_{\text {foot }}$ is the other radius of curvature. As a result, the neglect of hydrodynamic stresses near the bubble foot, leading to (4), yields

$$
-F_{2}^{\text {inertia }}-F_{2}^{\text {vort }}=\pi r_{\text {foot }}^{2}\left\{\left.\sigma\left(1 / R_{2}-1 / R_{1}\right)\right|_{\text {foot }}-\rho_{L} g h_{\text {wall }}\right\}+g \rho_{L} V_{b}+F_{2}^{\text {drag }} .
$$

This remarkable result shows that the sum of the two major force contributions, the overpressure force and the capillary force, is a directly measurable quantity. The difference of the radii of curvature at the bubble foot is equal to the square root of $4 H^{2}-K$, where $K$ is the Gaussian curvature. The difference can conveniently be determined from the measured contour and from

$$
1 / R_{2}-1 / R_{1}=((1))^{-1 / 2} R^{-1}\left\{R^{\prime} \operatorname{ctg}(\theta)-((0)) /((1))\right\} .
$$

Although the gravity term $-\rho_{L} g h_{\text {wall }}$ in (18) is usually less than $1 \%$ of the capillary term it accounts for much of the deformation of the interface, as can be observed by placing the chosen wall at various heights above the actual wall. The local values at the bubble foot in (18) are usually difficult to be measured accurately. For this reason another expression for $F_{2}{ }^{\Delta p}+F_{2}{ }^{\sigma}$ is now given.

Let the mean difference between the inverses of the radii of curvature be defined by

$$
\left\langle 1 / R_{2}-1 / R_{1}\right\rangle=\int_{0}^{\theta_{1}} d \theta R^{2}(\theta) \sin (\theta)\left(1 / R_{2}-1 / R_{1}\right) / \int_{0}^{\theta_{1}} d \theta R^{2}(\theta) \sin (\theta) .
$$

As long as $\Delta p=-\sigma\langle 2 H\rangle$ is a sufficient approximation of the pressure difference between the bubble and the liquid at the chosen wall, the sum of the two major force contributions can be obtained from

$$
F_{2}^{\Delta p}+F_{2}^{\sigma}=\pi r_{\text {foot }}^{2}\left\{\sigma\left\langle 1 / R_{2}-1 / R_{1}\right\rangle-\rho_{L} g h_{\text {wall }}\right\} .
$$

Because of the averaging over the gas-liquid interface above the chosen wall, (21) is expected to give more accurate results from measured bubble contours than the formally more accurate following expression:

$$
F_{2}^{\Delta p}+F_{2}^{\sigma}=\pi r_{\text {foot }}^{2}\left\{-\sigma\langle 2 H\rangle-\left(F_{1}^{\text {inertia }}+F_{1}^{g}+F_{1}^{d r a g}\right) /\left(\partial V_{b} / \partial b_{1}\right)-\rho_{L} g h_{\text {wall }}+2 \sigma /\left.R_{2}\right|_{\text {foot }}\right\} .
$$

Here, averaging $\langle 2 H\rangle$ is above the chosen wall and the radius of curvature $1 /\left.R_{2}\right|_{\text {foot }}$ can be determined from $-\sin \left(\theta_{c}\right) / r_{\text {foot }}$ or from

$$
1 / R_{2}=-((1))^{-1 / 2}\left\{1-R^{-1} R^{\prime} \operatorname{ctg}(\theta)\right\} .
$$

One of the main aims of the present study is to increase the measurement accuracy of force determinations. For this reason, the sum of the dominant forces $F_{2}{ }^{\Delta p}+F_{2}{ }^{\sigma}$ is assessed with the aid of (18), (21) and (22) and compared with "ideal" values $\left(\Delta p_{2} \pi r_{\text {foot }}{ }^{2}+F^{\sigma}{ }_{2}\right)$, where $\Delta p_{2}$ is the pressure drop that makes (17) an identity. 


\section{Hydrodynamic forces including drag}

The remaining force components of Eq. (2) that need to be quantified are $F_{j}^{\text {inertia }}, F_{j}{ }^{\text {vort }}$, and $F_{j}{ }^{d r a g}$. The inertia forces are given by the Lagrange equation

$$
\frac{d}{d t} \frac{\partial T^{\text {irrot }}}{\partial \dot{b}_{j}}-\frac{\partial T^{\text {irrot }}}{\partial b_{j}}=-F_{j}^{\text {inertia }} .
$$

The hydrodynamic force $F^{\text {inertia }}$ is computed from potential flow over truncated spheres. Up to this point in the analysis, general deformation of an axisymmetric bubble has been considered although the analysis was limited to the force balances corresponding to the first two generalized coordinated, $b_{1}$ and $h$. Since all three force components, $F_{j}^{\text {inertia }}, F_{j}^{\text {vort }}$, and $F_{j}^{\text {drag }}$, will be seen to have small contributions and since the experimentally observed shapes of bubbles are close to that of truncated spheres, inertia, and drag forces will only be determined for that of the truncated sphere closest to the actual shape. This means that the governing equations are given by

$$
\begin{aligned}
& -F_{1}^{\text {inertia }}\left(b_{1}, \dot{b}_{1}, \ddot{b}_{1}, b_{2}, \dot{b}_{2}, \ddot{b}_{2}\right)=F_{1}^{\text {vort }}\left(b_{1}, b_{1}, b_{2}, b_{2}\right)+F_{1}^{\Delta p}+F_{1}^{\sigma}+F_{1}^{g}+F_{1}^{\text {drag }}\left(b_{1}, b_{1}, b_{2}, b_{2}\right), \\
& -F_{2}^{\text {inertia }}\left(b_{2}, \dot{b}_{2}, \ddot{b}_{2}, b_{2}, \dot{b}_{2}, \ddot{b}_{2}\right)=F_{2}^{\text {vort }}\left(b_{1}, b_{1}, b_{2}, b_{2}\right)+F_{2}^{\Delta p}+F_{2}^{\sigma}+F_{2}^{g}+F_{2}^{\text {drag }}\left(b_{1}, b_{1}, b_{2}, b_{2}\right),
\end{aligned}
$$

where the forces $F^{\Delta p}, F^{\sigma}$, amd $F^{g}$ depend on all 25 generalized coordinates and are determined in ways presented in the above Sec. III B.

A bubble with the shape of a truncated sphere (distance $h$ and radius $R$ ) in a uniform flow field with velocity $V$ experiences a lift force resulting from the induced pressure field. This lift force is part of $F_{2}{ }^{\text {inertia }}$. To determine the inertia forces and viscous potential drag, the kinetic energy is written as a second order polynomial of the two prevailing generalized velocities

$$
T^{\text {irrot }} /\left(1 / 2 \rho_{L} V_{0}\right)=\alpha U^{2}+v \dot{R}_{\text {sphere }}^{2}+\psi \dot{R}_{\text {sphere }} U+\alpha_{2} V^{2} .
$$

Here, $V_{0}=\frac{4}{3} \pi R_{\text {sphere }}{ }^{3}$. Each coefficient occurring on the RHS of this expression is an added mass coefficient; coefficient $v$ was written as $\operatorname{tr}(\beta)$ in Ref. 4. Each coefficient depends on generalized coordinates but not on generalized velocities. The coefficient $\alpha$ is familiar and has the value 0.5 far away from the wall. Values of added mass coefficients have been obtained both for spheres and truncated spheres. ${ }^{7,4}$ In the present study, these added mass coefficients yield an estimate of $F^{\text {inertia }}$ by using $R_{\text {sphere }}$ and $h$ as parameters that characterize the truncated sphere with a shape closest to the actually measured bubble shape. Convenient expressions to evaluate the added mass coefficients are given in the Appendix.

Viscous drag forces for truncated spheres can be determined from the potential flow field by the Levich approach. ${ }^{18,4}$ Similar to the above determination of $F^{\text {inertia }}$, drag force $F^{\text {drag }}$ is overestimated with the aid of measured parameters $R_{\text {sphere }}$ and $h$ that characterize the truncated sphere shape closest to the actual bubble. The accuracy of the inertial lift and viscous potential drag will be discussed below. In the following equations, parameter $R_{\text {sphere }}$ is written as $R$ for sake of brevity and $U$ is written as $\dot{h}$ to highlight similarity in the equations with respect to $\dot{R}$ and $\dot{h}$. The Euler-Lagrange equation for the first generalized coordinate, $R_{\text {sphere }}$, yields

$$
\frac{4}{3} \pi \rho_{L} R_{0}^{3}\left(1 / 2 \psi \ddot{h}+v \ddot{R}+M_{1}\right)=F_{1}^{v o r t}+F_{1}^{\Delta p}+F_{1}^{\sigma}+F_{1}^{g}-W_{11} \dot{R}-W_{12} \dot{h}
$$

$\left(R_{0}=R(t=0)\right.$, the initial value of $\left.b_{1}\right)$ with inertia lift given by

$$
M_{1}=\frac{1}{2} \dot{R}^{2}\left(\frac{\partial v}{\partial R}+3 v / R\right)-\frac{1}{2} \dot{h}^{2}\left(\frac{\partial \alpha}{\partial R}-\frac{\partial \psi}{\partial h}+3 \alpha / R\right)+\dot{h} \dot{R} \frac{\partial v}{\partial h}+\frac{1}{2} V^{2}\left(\frac{\partial \alpha_{2}}{\partial R}+3 \alpha_{2} / R\right) .
$$

The Euler-Lagrange equation for $h$ renders the second governing equation to the form

$$
\frac{4}{3} \pi \rho_{L} R_{0}^{3}\left(\alpha \ddot{h}+1 / 2 \psi \ddot{R}+M_{2}\right)=F_{2}^{\text {vort }}+F_{E}^{\Delta p}+F_{2}^{\sigma}+V_{b} g \rho_{L}-W_{21} \dot{R}-W_{22} \dot{h},
$$

with inertia lift given by

$$
M_{2}=\frac{1}{2} \dot{R}^{2}\left(\frac{\partial \psi}{\partial R}-\frac{\partial v}{\partial h}+3 \psi / R\right)+\frac{1}{2} \dot{h}^{2} \frac{\partial \alpha}{\partial h}+\dot{h} \dot{R}\left(\frac{\partial \alpha}{\partial R}+3 \alpha / R\right)-\frac{1}{2} V^{2} \frac{\partial \alpha_{2}}{\partial h} .
$$


Coefficients $W_{i j}$ are drag coefficients which only depend on the generalized coordinates $\left(R=R_{\text {sphere }}\right.$ and $h$ ). For a sphere far away from the wall, both $W_{11}$ and $W_{22}$ reduce $^{4,7}$ to $12 \pi \mu R_{\text {sphere. In Ref. }}$ 7 , it was shown that literature results for the added masses for a touching sphere $\left(\lambda=\frac{1}{2}\right)$ and for either $\dot{R}=0$ or $\dot{R}=U$ are in agreement with those obtained with the above approach. Convenient expressions to evaluate $W_{11}$ and $W_{22}$ for a truncated sphere at a plane wall are given in the Appendix. In the measurements of this study, bubble shapes are found to be close to those of truncated spheres. The range of applicability and the accuracy of the inertial lift and viscous potential drag for the measurements of this study will be discussed in Sec. III E below.

\section{Dimensionless form of the governing equations}

The governing equations can be written in dimensionless form by dividing the dimensional equations by $\rho_{L} R_{0}^{2} \dot{R}_{0}^{2}$, where $\dot{R}_{0}$ is defined as the time rate of change of $b_{1}$ at initial time zero, $\dot{b}_{1}(t=0), \dot{h}_{0}$ is that of $h$ at time zero, and $R_{0}$ is the initial value of $b_{1}$. In boiling, $\dot{R}_{0}$ is bound to be nonzero and positive which makes it a good reference velocity. The velocities $\dot{h}$ and $\dot{R}=\dot{b}_{1}$ are coupled by the governing equations, but velocity of the approaching flow at the center of the bubble, $V$, is an independent velocity. Three Reynolds numbers exist:

$$
R e_{1}=\dot{R}_{0} R_{0} \rho_{L} / \mu, \quad R e_{2}=\dot{h}_{0} R_{0} \rho_{L} / \mu, \quad R e_{V}=V R_{0} \rho_{L} / \mu
$$

Other dimensionless numbers are the Weber, Froude and dimensionless pressure numbers which are, respectively, defined by

$$
W e=\rho_{L} R_{0} \dot{R}_{0}^{2} / \sigma, \quad F r=\dot{R}_{0}^{2} /\left(g R_{0}\right), \quad \Delta P_{0}=\Delta P(t=0) /\left(\rho_{L} \dot{R}_{0}^{2}\right) .
$$

With $W_{i j}=\hat{W}_{i j} b_{1} \pi \mu$, the dimensionless governing equation corresponding to $h$ becomes

$$
\begin{aligned}
\frac{4}{3} \pi & R_{0}\left(\left(\operatorname{Re}_{2} / \operatorname{Re}_{1}\right)^{2} \alpha \ddot{h} / h_{0}^{2}+1 / 2 \psi \ddot{R} / \dot{R}_{0}^{2}\right)=-\frac{4}{3} \pi \breve{M}_{2}+\breve{F}_{2}^{\text {vort }}+\pi\left(r_{\text {foot }}^{2} / R_{0}^{2}\right) \Delta P_{0}-2 \pi \\
& \sin \left(\theta_{c}\right)\left(r_{\text {foot }} / R_{0}\right) / W e+\frac{4}{3} \pi\left(V_{b} / V_{0}\right) / F r-\hat{W}_{22} \pi\left(b_{1} / R_{0}\right)\left(\dot{h} / \dot{h}_{0}\right)\left(R e_{2} / R e_{1}\right) / R e_{1}- \\
& \hat{W}_{21} \pi\left(b_{1} / R_{0}\right)\left(\dot{b}_{1} / \dot{R}_{0}\right) / \operatorname{Re}_{1}
\end{aligned}
$$

with the inertia forces given by

$$
\begin{aligned}
\breve{M}_{2}= & 1 / 2\left(R_{0} \frac{\partial \psi}{\partial b_{1}}-R_{0} \frac{\partial v}{\partial h}+3 \psi R_{0} / R\right)\left(\dot{b}_{1} / \dot{R}_{0}\right)^{2}+1 / 2\left(R e_{2} / R e_{1}\right)^{2}\left(\dot{h} / \dot{h}_{0}\right)^{2} R_{0} \frac{\partial \alpha}{\partial h}+\left(R e_{2} / R e_{1}\right) \\
& \left(\dot{h} / \dot{h}_{0}\right)\left(\dot{b}_{1} / \dot{R}_{0}\right)\left(R_{0} \frac{\partial \alpha}{\partial b_{1}}+3 \alpha R_{0} / R\right)-1 / 2 R_{0} \frac{\partial \alpha_{2}}{\partial h}\left(R e_{V} / R e_{1}\right)^{2} .
\end{aligned}
$$

Dimensionless vorticity force, $\tilde{F}_{2}^{\text {vort }}$, equals $F_{2}^{\text {vort }} /\left(\rho_{L} R_{0}^{2} \dot{R}_{0}^{2}\right)$. Note that $\left(\dot{h} / \dot{h}_{0}\right),\left(\dot{b}_{1} / \dot{R}_{0}\right)$ and $\left(b_{1} / R_{0}\right)$ are initially 1 while both $\left(R e_{2} / R_{1}\right)$ and $\left(V_{b} / V_{0}\right)$ are of order 1 .

These expressions not only make clear how the Reynolds numbers determine the importance of viscous Levich drag, as usual, but also that capillary forces are controlled by the Weber number and body forces by the Froude number. The pressure term with dimensionless pressure $\Delta P_{0}$ is usually the source of high-frequency oscillations, depending on the equation of state of the gas in the bubble. ${ }^{8}$ The gradients of added mass coefficients, such as $\frac{\partial \alpha}{\partial h}$, are also familiar contributions. ${ }^{7,9}$ The various inertia forces depend on two Reynolds number ratios. The last contribution, with $\left(R e_{V} / R e_{1}\right){ }^{2}$ is the familiar inertia lift due to uniform approaching flow. Note that $\frac{\partial \alpha_{2}}{\partial h}$ is positive which implies that this lift force pushes the bubble away from the wall. In the experiments of this study, the Froude number is small which makes the contribution of the Froude term to the force balance less important. In addition, in the experiments of this study the Reynolds number $R_{1}$ is of order 1 which renders the contribution of the drag components to the force balance more important than in high-Reynolds number experiments, which are more common. It will be shown in Sec. III E that the viscous potential drag yields overestimations of the actual drag. If the drag contributions will nevertheless be found to be negligible it can safely be assumed that drag plays a negligible role in bubble growth in the boiling conditions of the present study (low Ja-number). 
In the same manner the extended Rayleigh-Plesset equation for a bubble attached to a plane wall can be given the following dimensionless form:

$$
\begin{gathered}
\frac{4}{3} \pi R_{0}\left(v \ddot{R} / \dot{R}+{ }^{1} / 2\left(R e_{2} / R e_{E}\right)^{2} \psi \ddot{h} / \dot{h}_{0}^{2}\right)=-\frac{4}{3} \pi \breve{M}_{1}+\breve{F}_{1}^{\text {vort }}+2 \pi(1-ю)\left\langle R^{2}\right\rangle R_{0}^{-2} \Delta P_{0} \\
-2 \pi(1-ю)\left\langle R^{2}\right\rangle R_{0}^{-1}\langle 2 H\rangle / W e+2 \pi(1-ю)\left(h\left\langle R^{2}\right\rangle+\left\langle x R^{3}\right\rangle\right) R_{0}^{-3} / F r-\hat{W}_{11} \\
\pi\left(b_{1} / R_{0}\right)\left(\dot{b}_{1} / \dot{R}_{0}\right) / R e_{1}-\hat{W}_{21} \pi\left(b_{1} / R_{0}\right)\left(\dot{h} / \dot{h}_{0}\right)\left(\operatorname{Re}_{2} / \operatorname{Re}_{1}\right) / R e_{1}
\end{gathered}
$$

with the inertia forces given by

$$
\begin{gathered}
\breve{M}_{1}=1 / 2\left(R_{0} \frac{\partial v}{\partial b_{1}} 3 v R_{0} / R\right)\left(\dot{b}_{1} / \dot{R}_{0}\right)-1 / 2\left(R e_{2} / R e_{1}\right)^{2}\left(\dot{h} / \dot{h}_{0}\right)^{2}\left(R_{0} \frac{\partial \alpha}{\partial b_{1}}-R_{0} \frac{\partial \psi}{\partial h}+3 \alpha R_{0} /\right. \\
R)+\left(R e_{2} / R e_{1}\right)\left(\dot{b}_{1} / \dot{R}_{0}\right)\left(\dot{h} / \dot{h}_{0}\right) R_{0} \frac{\partial v}{\partial h}+1 / 2\left(R_{0} \frac{\partial \alpha_{2}}{\partial R} 3 \alpha_{2} R_{0} / R\right)\left(R e_{V} / R e_{1}\right)^{2} .
\end{gathered}
$$

The two governing equations are clearly two coupled differential equations of the same kind. Note that the factors multiplying the dimensionless numbers $W e, F r$, and $\Delta P_{0}$ are different in the two dimensionless equations above. The terms with $W e, F r$, and $\Delta P_{0}$ are applicable to any case of axisymmetric bubble deformation. The other terms, the inertia and drag terms, are exact for truncated spheres and take slightly different values in the case of more general deformation. ${ }^{8}$ With general deformation, each of the added mass and drag coefficients used above depends on all generalized coordinates; for example: $v=v\left(b_{1}, h, b_{2}, b_{3}, b_{4}, \ldots\right)$. In the case of a truncated sphere, the added mass coefficients turn out ${ }^{9}$ to be dependent on only one geometrical parameter, $\lambda=b_{1} /(2 h)$. The Appendix gives convenient fit functions that describe dependencies of $\alpha, v, \psi, \alpha_{2}$ and $W_{11}, W_{12}$, $W_{22}$ on $\lambda$.

\section{E. Range of applicability of derived drag and lift forces for boiling bubbles}

Two facts are often overlooked in estimating the lift force on a boiling bubble:

- The inertial contribution to the lift, i.e., those corresponding to the potential flow part of the flow, prevail at small Reynolds numbers.

- There is a finite time for vorticity generation and built-up of boundary layers and the domain where vorticity may occur is decreasing in the course of time.

The validity of added mass coefficients computed for flows at low or intermediate Reynolds number has been discussed in the above. ${ }^{4,15}$ As a result of their general validity, all terms computed from added mass coefficients and collected in $M_{2}$ contribute to temporal changes in distance $h$ of the center of the bubble to the wall, whatever the Reynolds number. Traditionally, often only the term related to the uniform approach velocity, $V$, is denoted with "lift force," but all added-mass terms contribute to motion of the bubble center away from or towards the wall. We therefore prefer to group inertia forces not involving generalized accelerations $\left(M_{2}\right)$ and name them the potential flow lift on the bubble. This grouping avoid the naming of individual terms of the potential lift, such as "unsteady growth force" 12,13 and "hydrodynamic force." 12 Whatever the Reynolds number, potential lift occurs and the only lift force contribution missing in our analysis stems from the vorticity contribution $F_{2}{ }^{\text {vort }}$. At high-Reynolds number flows, only potential lift is important since the vorticity is confined to thin boundary layers with a thickness inversely proportional to the square root of the Reynolds number. ${ }^{14}$ In previous publications, ${ }^{7,8}$ it has been shown that well-known results for high-Reynolds number flow are consistent with the present computations of inertia forces. The finite time of existence, the second point above, is important for the estimation of $F_{2}{ }^{\text {vort }}$ which will be discussed after a digression about the drag force.

It is instructive to combine the information concerning the drag force coefficient of a hemisphere in uniform flow at a plane wall ${ }^{19}$ for $R e_{V}>0.1$ with the available information concerning the time dependency of the drag coefficient for a sphere with a growing radius ${ }^{20}$ in an effort

- to elucidate the competition of diffusion time scale with time of growth and

- to estimate the errors of the estimates of lift and drag employed in this study. 
A good fit of the $C_{D}$-data of the hemisphere in quasi-steady conditions is given ${ }^{19}$ by

$$
C_{D}=\left(48 / \operatorname{Re}_{V}\right)\left\{1-(5 / 12)\left(1-\tanh \left(\operatorname{Re}_{V} / 70\right)\right)\right\},
$$

where the term proportional to (5/12) goes to zero in the limit of $R e_{V}$ going to infinity. The asymptotic limit of $C_{D}$ is already practically reached at $R e_{V}=200$. With decreasing $R e_{V}$ the drag force coefficient decreases from $48 / R e_{V}$ to $28 / R e_{V}$ since $1-5 / 12=28 / 48$. The reason for this decrease is the gathering of vorticity especially at places where the potential flow solution predicts large velocity gradients, i.e., near the foot of the hemisphere. Pressure differences are reduced within these regions and in addition the energy dissipation is reduced at these locations. The resulting drag reduction explains the minus sign in the second term of the above expression. For the same reason the drag of a full sphere is reduced ${ }^{14}$ from $48 / R e_{V}$ to $\left(48 / R e_{V}\right)\left(1-2.2 / R e_{V}{ }^{0.5}\right)$.

If a sphere with radius $R_{0}$ is at time zero suddenly put in a uniform flow with velocity $V$, and if the sphere grows in time according to $R(t)$ (a situation closely resembling the boiling bubbles of the experiments here reported, except for the shape), the drag force is in the course of time given ${ }^{20}$ by

$$
-F_{D}=12 \pi \mu R(t) V+8 \pi \mu\left(-R(t) V+R_{0} V f_{1}(t)+R(t) V f_{2}(t)\right) .
$$

At time zero, $f_{1}$ equals 1 and $f_{2}$ is zero, making the second term on the RHS of the above expression equal to zero. Whatever the Reynolds number, initially the drag force is given by the viscous potential drag of the first term on the RHS. ${ }^{21}$ The viscous dimensionless time scale $v t R_{0}^{-2}$ is replaced by ${ }_{0} \int^{t} v R(t)^{-2} d t$. If the derivative of the radius with respect to time is nonzero, the $f_{2}$-term is a nonzero history force contribution. If this derivative is positive, as for our boiling bubbles, also the $f_{2}$-term is positive. The limit for $t$ going to infinity of both $f_{1}$ and $f_{2}$ is zero, making the quasi-steady drag equal to $4 \pi \mu R V$. For sake of completeness, the functions $f_{1}$ and $f_{2}$ for a sphere are given ${ }^{20}$ by $\left(v=\mu / \rho_{L}\right)$,

$$
\begin{gathered}
f_{1}(t)=\exp \left(9 v \int_{0}^{t} R\left(t^{\prime}\right)^{-2} d t^{\prime}\right) \operatorname{erfc}\left(\left\{9 v \int_{0}^{t} R\left(t^{\prime}\right)^{-2} d t^{\prime}\right\}^{0.5}\right), \\
f_{2}(t)=(1 / R(t)) \int_{0}^{t} \exp \left(9 v \int_{\tau}^{t} R\left(t^{\prime}\right)^{-2} d t^{\prime}\right) \operatorname{erfc}\left(\left\{9 v \int_{\tau}^{t} R\left(t^{\prime}\right)^{-2} d t^{\prime}\right\}^{0.5}\right) \frac{\partial R(\tau)}{\partial \tau} d \tau .
\end{gathered}
$$

For $\operatorname{Re}_{V}<1$, at large times the kernel of the memory integral corresponding to the history force is not of the above form any more. This is because far from the particle, the advection terms neglected in the derivation of $f_{1}$ and $f_{2}$ become significant in the transport of the vorticity field. ${ }^{22}$ Application is therefore recommended only for $\operatorname{Re}_{V} \geq 1$.

If $R e_{1} \gg 1$, Reynolds number $R e_{1}$ is the relevant ratio of inertia to viscous effects involved, and drag may be ${ }^{20}$ close to the viscous potential drag whatever the value of $\operatorname{Re}_{V}$. The time scale corresponding to bubble expansion is in competition with the diffusion time scale and the quasisteady viscous drag force is therefore an underestimation of the actual drag on a growing boiling bubble. For a growing hemisphere at a plane wall the same competition occurs but the quasi-steady drag is different. We therefore postulate that the time dependent drag on is in this case approximately represented

$$
-F_{D}=12 \pi \mu R(t) V+5 \pi \mu V\left(1-\tanh \left(R e_{V} / 70\right)\right)\left(-R(t)+R_{0} f_{1}(t)+R(t) f_{2}(t)\right),
$$

where $f_{1}$ and $f_{2}$ are in principle different from those given above but with the same asymptotic behavior. When the growth rate of the hemisphere is small and the diffusion time scale dominates the expansion time scale, the above expression reproduces the correct value of $12 \pi \mu$ $R(t) V\left\{1-(5 / 12)\left(1-\tanh \left(\operatorname{Re}_{V} / 70\right)\right)\right\}$. When bubble growth is fast and/or the lifetime of the bubble is short, the correct asymptotic value given by viscous potential drag, $12 \pi \mu R(t) V$, is reproduced. The above expression is expected to be a reasonable approximation of the drag force on a hemisphere for $R e_{1} \geq 1$ and $R e_{V} \geq 1$.

The above drag acts in a direction parallel to the approaching flow. In the present study, drag perpendicular to the wall, connected to $W_{22}$, and drag opposing bubble expansion, connected to $W_{11}$, as well as a mixed term, connected to $W_{12}$, is computed. Both $W_{11}$ and $W_{22}$ have the asymptotic 
value of $12 \pi \mu R(t)$ for a sphere in an unbounded fluid. We may therefore postulate that the drag on a growing truncated sphere at a plane wall is approximately represented by an equation of the form

$$
\begin{aligned}
& -F_{11}^{\text {drag }}=W_{11} \dot{b}_{1}+W_{11} \dot{b}_{1}(5 / 12)\left(1-\tanh \left(R e_{V} / 70\right)\right)\left(-1+\left(R_{0} / R(t)\right) f_{1}(t)+f_{2}(t)\right), \\
& -F_{22}^{\text {drag }}=W_{22} \dot{h}+W_{22} \dot{h}(5 / 12)\left(1-\tanh \left(\operatorname{Re}_{V} / 70\right)\right)\left(-1+\left(R_{0} / R(t)\right) f_{1}(t)+f_{2}(t)\right),
\end{aligned}
$$

where once again functions $f_{1}$ and $f_{2}$ may depend on the shape at hand but possess the same asymptotic behavior as in (36a) and (36b). As before, asymptotic values for $R e_{1}$ and $R e_{2}$ going to infinity are correct if the viscous potential drag terms, $W_{11}$ and $W_{22}$, are computed for the shape of the truncated sphere at hand. There is no equivalent of a drag force connected to $W_{12}$ in the literature, but we may expect a similar expression to hold for this drag contribution; $F_{1}^{\text {drag }}=F_{11}^{\text {drag }}+F_{12}^{\text {drag }}$; $F_{2}^{\text {drag }}=F_{22}^{\text {drag }}+F_{12}^{\text {drag }}$.

The main conclusions to be gathered from these drag force expressions are

1. the faster bubble growth or the shorter the lifetime of a boiling bubble, the more close actual drag is to viscous potential drag;

2. the presence of a plane wall increases the quasi-steady drag on a hemisphere as compared to that of a sphere, and a similar increase is expected for bubbles with the shape of a truncated sphere;

3. if anything, the potential drag yields an overestimation of the actual drag for a boiling bubble with a deviation that can be estimated with the aid of the above equations.

Typical times of growth that have been observed in our experiments up to detachment from the wall is for microgravity bubbles $0.03 \mathrm{~s}$ (radius grows from 0.05 to $0.09 \mathrm{~mm}$ ) and for terrestrial ones $0.05 \mathrm{~s}$ (radius grows from 0.12 to $0.19 \mathrm{~mm}$ ). With the aid of the above expression for $-F_{22}^{\text {drag }}$ and the $f_{1}$ and $f_{2}$ functions of a sphere given by (36a) and (36b), the drag $W_{22} \dot{h}$ is computed to be reduced to about $0.73 W_{22} \dot{h}$ (microgravity) and to about $0.76 W_{22} \dot{h}$ (terrestrial) at the end of the time of growth. These values 0.73 and 0.76 required the computation of the integrals in (36a) and (36b), of course. In the remainder of this study, computations will be made with viscous potential drag only, so, for example, with $-F_{22}^{\text {drag }}=W_{22} \dot{b}_{1}$, in the expectation that despite the overestimation drag will be found to be negligible. This expectation shall a posteriori be validated. If it turns out not to be fully satisfied, the maximum error occurring at the end of the growth time is estimated, on basis of the above computations, to be about $25 \%$. Making allowance for the effect of advection of vorticity by the approaching flow, which invalidates the assumption of a uniform approaching flow, the maximum error in the viscous drag estimate of drag is estimated to about $40 \%$. This error occurs at the time the bubble starts leaving the artificial nucleation site and is less at previous times. The same error is assumed for $W_{11}$ and $W_{12}$-contributions. Even though this is a rough estimate, it is sufficient in our conservative approach with a posteriori validation of the expectation that drag and lift contributions are negligible.

The building up of a vorticity layer in the vicinity of the liquid-vapor interface affects lift in a similar manner. In quasi-steady flow over a hemisphere at a plane wall and for $R e_{V}$ exceeding 1 , the lift force coefficient has recently been asssesed. ${ }^{19}$ After some algebraic manipulation, the lift coefficient turns out to be represented by

$$
C_{L}=(11 / 8)\left\{1-\left(0.3+0.0232 R e_{V}^{0.5}\right) /\left(1+0.02 R e_{V}\right)\right\},
$$

where $11 / 8$ is the potential flow solution for the inertia lift corresponding to approach velocity $V$. There is no account of the effect of the time-dependency of the bubble radius on lift in the literature. In analogy to the drag force treatment we therefore postulate for the hemisphere with radius $R(t)$ that in first order approximation

$$
\begin{aligned}
F_{2}^{l i f t}= & \left.1 / 4(11 / 8) \pi \rho R(t)^{2} V^{2}+1 / 4(11 / 8) \pi \rho R(t)^{2} V^{2}\left(\left(0.3+0.0232 R e_{V}^{0.5}\right) / 1+0.02 R e_{V}\right)\right) \\
& \times\left(-1+\left(R_{0} / R(t)\right) f_{1}(t)+f_{2}(t)\right) .
\end{aligned}
$$


For an expanding bubble with the shape of a truncated sphere the $V^{2}$-lift becomes

$$
\begin{aligned}
F_{2}^{l i f t}= & 2 / 3 \pi \rho \frac{\partial \alpha_{2}}{\partial h} R(t)^{3} V^{2}+2 / 3 \pi \rho \frac{\partial \alpha_{2}}{\partial h} R(t)^{3} V^{2}\left(\left(0.3+0.0232 R e_{V}^{0.5}\right) /\left(1+0.02 R e_{V}\right)\right) \\
& \times\left(-1+\left(R_{0} / R(t)\right) f_{1}(t)+f_{2}(t)\right) .
\end{aligned}
$$

Despite the fact that the time-dependent functions $f_{1}$ and $f_{2}$ may differ from the functions $f_{1}$ and $f_{2}$ given above,$^{20}$ the trends predicted by the latter functions will be the same. Similarly, the low-Reynolds number correction factor $\left(\left(0.3+0.0232 \operatorname{Re}_{V}{ }^{0.5}\right) /\left(1+0.02 R e_{V}\right)\right)$ may be somewhat different for shapes deviating from that of a hemisphere, the trend in the $R e_{V}$-dependency predicted will be the same. The benefit of the above expression is therefore that it predicts the correct limiting values and the proper trends, in particular, the tendency of vorticity to decrease the lifting action of the potential flow. ${ }^{19}$ The reason for this action is that pressure differences are reduced within the regions where vorticity is gathered, as mentioned above, leading to a reduced pressure difference between bubble foot and top of the bubble. Lift is the pressure difference integrated over the bubble surface, and is therefore also decreased with increasing importance of vorticity. In the remainder of this study, computations will be made with inertia lift $2 / 3 \pi \rho \frac{\partial \alpha_{2}}{\partial h} R(t)^{3} V^{2}$ in the expectation that despite the overestimation lift will be found to be negligible. This expectation shall a posteriori be validated.

Terms in the inertia lifts $M_{1}$ and $M_{2}$ other than those proportional to $V^{2}$, i.e., terms with $\frac{\partial v}{\partial h}$, for example, are pure inertia terms and unaffected by the presence of vorticity. They retain their value whatever the values of the three Reynolds numbers. ${ }^{4,15}$

To the best of our knowledge, the expressions given above are the most appropriate ones for the terrestrial and microgravity bubbles measured in our experiments. However, other expressions for $F^{\text {vort }}$ and $F^{d r a g}$ could easily be combined with the expressions for $F^{\text {inertia }}, F^{\Delta p}, F^{\sigma}$, and $F^{g}$ given above, following Eq. (2). In addition, more complex deformation is easily accommodated by taking all (25) generalized coordinates into account in the assessment of $F_{2}{ }^{\text {inertia }}\left(b_{j}, \dot{b}_{j}, \ddot{b}_{j}\right), F_{2}{ }^{\text {vort }}\left(b_{j}, \dot{b}_{j}\right)$, and $F_{2}{ }^{d r a g}\left(b_{j}, \dot{b}_{j}\right)$. The other forces already take account of these full dependencies in the present study. The expressions for drag and lift given above are recommended for growing boiling bubbles if either $R e_{1} \gg 1$ or $R e_{2} \gg 1$, or if both $R e_{1} \geq 1$ and $R e_{2} \geq 1$ and if either $R e_{V} \gg 1$ or the lifetime of bubbles is short.

\section{RESULTS}

\section{A. Effect of (micro)gravity on heat transfer}

More heating power has been required to create boiling bubbles in terrestrial gravity than in microgravity. In our experiments, bubbles grow slower in microgravity. Since subcooling and velocity of the approaching fluid flow have been the same in the terrestrial and microgravity experiments, the necessity of a higher heating power on the ground is probably due to enhancement of heat transfer by natural convection. Typical bulk Reynolds number is 330, at approach velocity $1.2 \mathrm{~cm} / \mathrm{s}$, and the boundary layer at distance $0.493 \mathrm{~mm}$ from the frontal edge of the bubble generator is laminar (kinematic viscosity $v_{L}=3.2 \times 10^{-7} \mathrm{~m}^{2} / \mathrm{s}$ ). The velocity boundary layer thickness is there estimated $^{23}$ as the square root of $\left((1260 / 37) v_{L} 0.493 \times 10^{-3} / V\right)$, which is $0.6 \mathrm{~mm}$. The temperature boundary layer thickness is less, $0.24 \mathrm{~mm}$, because of the Prantl number of 7.8. Bubbles grow in the boundary layer. Since bubbles are fully embedded in the thermal plume over the heater, bubble growth evolution is to sufficient degree described ${ }^{24}$ by

$$
R(t)=J a \sqrt{\left(a_{L} t\right)},
$$

where $a_{L}=\lambda_{L} /\left(\rho_{L} c_{p, L}\right)$, the heat diffusivity of the liquid (typically $4.121 \times 10^{-8} \mathrm{~m} / \mathrm{s}^{2}, \lambda_{L}$ is heat conductivity of the liquid and $c_{p, L}$ its heat capacity, $\rho_{V}$ is mass density of the vapor), and the Jacob number depends on the vaporization enthalpy, $\Delta H$ (typically $1.42 \times 10^{5} \mathrm{~J} / \mathrm{kg}$ ): $J a=\rho_{L} c_{p, L}$ $\left(T_{w}-T_{s a t}\right) /\left(\rho_{V} \Delta H\right)$. Saturation temperature is denoted as $T_{s a t}$ and the temperature of the wall as $T_{w}$. The radius in the above equation has to be the volume-equivalent radius. The histories of the 


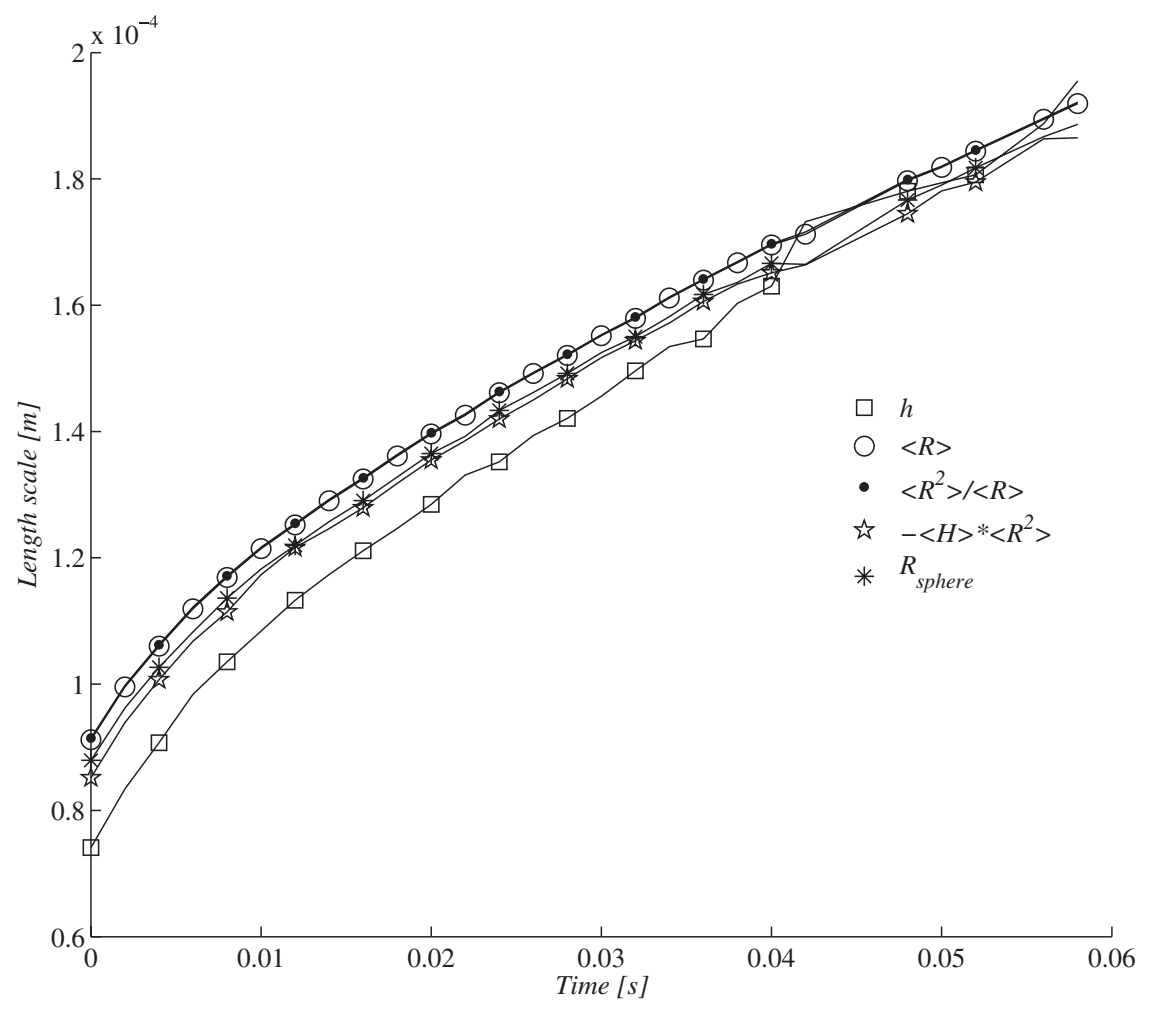

FIG. 8. Comparison of length scale histories for a terrestrial bubble.

volume-equivalent radii of 12 bubbles have been fitted with high accuracy to $J a \sqrt{ }\left(a_{L}\left(t-t_{0}\right)\right)$ with $t_{0}$ the time of growth before the first observation of the bubble with the camera. With $95 \%$ confidence interval the results of all bubbles are given by $\left(\Delta T=T_{w}-T_{s a t}\right)$,

terrestrial : $\quad J a=3.22 \pm 0.13 ; \Delta T=2.00 \pm 0.08{ }^{\circ} \mathrm{C} ; t_{\text {leave }}=0.088 \pm 0.006 \mathrm{~s}$, microgravity : $\quad J a=1.92 \pm 0.13 ; \Delta T=1.19 \pm 0.08{ }^{\circ} \mathrm{C} ; t_{\text {leave }}=0.055 \pm 0.02 \mathrm{~s}$.

The time of leaving the nucleation site, $t_{\text {leave, }}$, is in the terrestrial case equal to the time of detachment but has in the microgravity case to be estimated from the observations as the time the bubble starts leaving the site by moving parallel to the wall in downstream direction. Bearing in mind that subcooling of the approaching liquid is $4{ }^{\circ} \mathrm{C}$, a total temperature difference of about $6^{\circ}$ has to be overcome by the power to the bubble generator in the terrestrial case.

Froude number, equal to $R e^{2} / G r$, is for a typical length scale of $5 \mathrm{~mm}$ and typical driving temperature differences of 5 and $6 \mathrm{~K}$ estimated to be about 0.7 in on-ground experiments and about 154 in microgravity experiments at minimum ( $g$-jitter of $0.045 \mathrm{~m} / \mathrm{s}^{2}$ at maximum; expansion coefficient $\left.2.1910^{-3} 1 / \mathrm{K}\right)$. Boundary layer development is therefore affected by mixed convection in on-ground measurements, but merely by forced convection in microgravity.

On ground, the Jacob number is highest and bubble growth is more rapid. This is confirmed by time histories of length scales measured, as those given by Fig. 8. Notice that at later times in this bubble growth history the shape is not that of a truncated sphere as distance $h$ exceeds radius $R_{\text {sphere }}$. Since heat transfer rate in terrestrial conditions is higher because of natural convection, a higher superheating of the wall is required to create boiling. It is therefore hard to get identical bubble growth histories in terrestrial and microgravity conditions. The present study applies a force balance analysis to assess the major agents involved and to allow for a prediction of forces in case bubble growth rates in microgravity would be as high as on ground. This analysis is presented in Secs. IV B-IV F. 


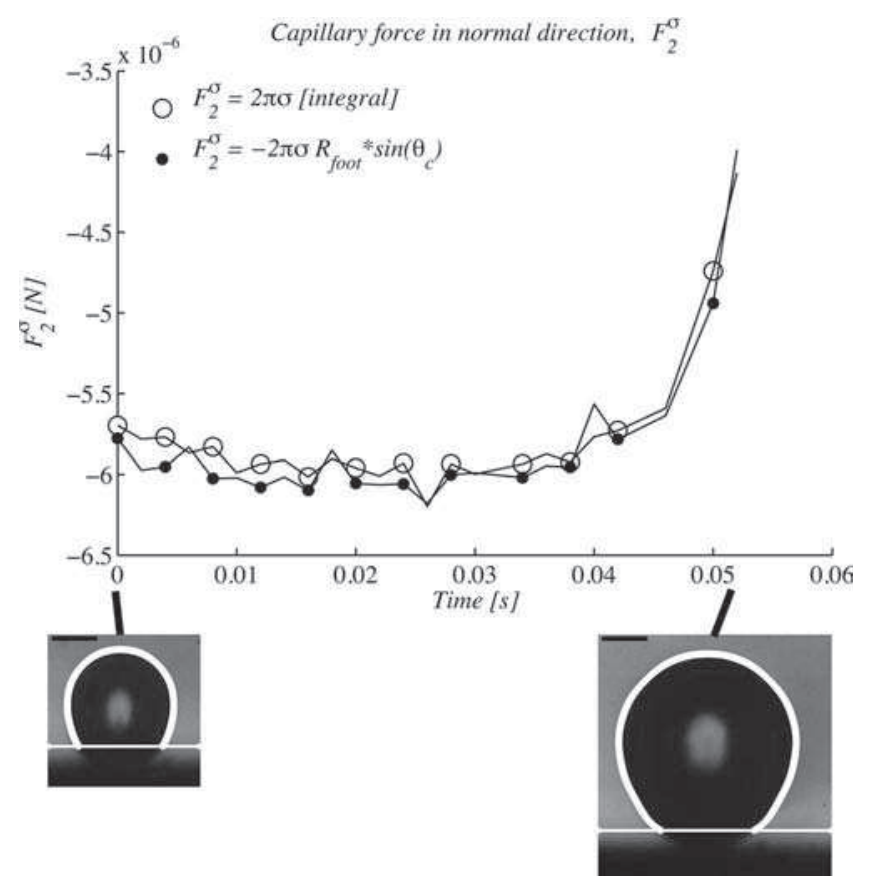

FIG. 9. Comparison of histories of $F^{\sigma} 2$ computed in two ways, one with the aid of the contact angle $\theta_{c}$ and (11) and the other with the integral equation (10).

The mean detachment radius, $R_{\text {leave }}$, for the two body force conditions is given by

terrestrial : $\quad R_{\text {leave }}=0.1945 \pm 0.0018 \mathrm{~mm}$, contact angle $38^{\circ} \pm 4^{\circ}$; microgravity : $\quad R_{\text {leave }}=0.091 \pm 0.016$.

Development of an improved criterion for the prediction of the bubble radius at time of leaving the nucleation site is beyond the scope of the present investigation.

\section{B. Assessment of accuracies of various methods to determine force components}

The important force that attracts a bubble to the wall is the capillary force $F_{2}^{\sigma}$. Figure 9 shows that the integral equation (10) yields about the same values as (11) for a low chosen wall. For a higher chosen wall differences are negligible because of the improved accuracy of the contact angle $\theta_{c}$ necessary for (11). The decrease in absolute value of the attracting capillary force with increase of time in later stages of bubble growth is easily understood from the decrease in apparent contact angle and from (11). The apparent contact angle decreases from about $60^{\circ}$ to about $40^{\circ}$ in the course of time. The variation in the course of time of the capillary force is about the same in both ways of computing it. The accuracy of both ways is expected to be the same.

The $b_{1}$-equation for a bubble attached to a plane wall, the first Euler-Lagrange equation (16), can be considered as an extended Rayleigh-Plesset equation valid for bubbles of any shape. At each instant of time it contains only one unknown, the overpressure $\Delta p$. Figure 10 shows the time history of the resulting values of the overpressure, at each instant of time given by $\Delta p=-\sigma\langle 2 H\rangle-\left(F_{1}{ }^{\text {inertia }}\right.$ $\left.+F_{1}{ }^{g}+F_{1}{ }^{\text {rag }}\right) /\left(\partial V_{b} / \partial b_{1}\right)$. The approximation $\Delta p=-\sigma\langle 2 H\rangle$ is found to be very close at all times, as expected. Discrepancies are primarily due to the gravity term $F_{1}{ }^{g}$. Drag is overestimated but turns out to be negligible and inertial lift is contributing in later stage of bubble growth, but only little. Although this lift is estimated, using expressions only valid for a truncated sphere, the estimates are sufficiently well to be able to state that the overpressure depends merely on the mean curvature term, $-\sigma\langle 2 H\rangle$, and on gravity which is measured via the measured bubble volume. The definition-based approximation $\Delta p=-\left.2 \sigma H\right|_{\text {foot }}-\rho_{L} g h_{\text {wall }}$ of (4) depends on the accuracy of the measurement of the bubble foot and is found to vary less smoothly in the course of time than the 


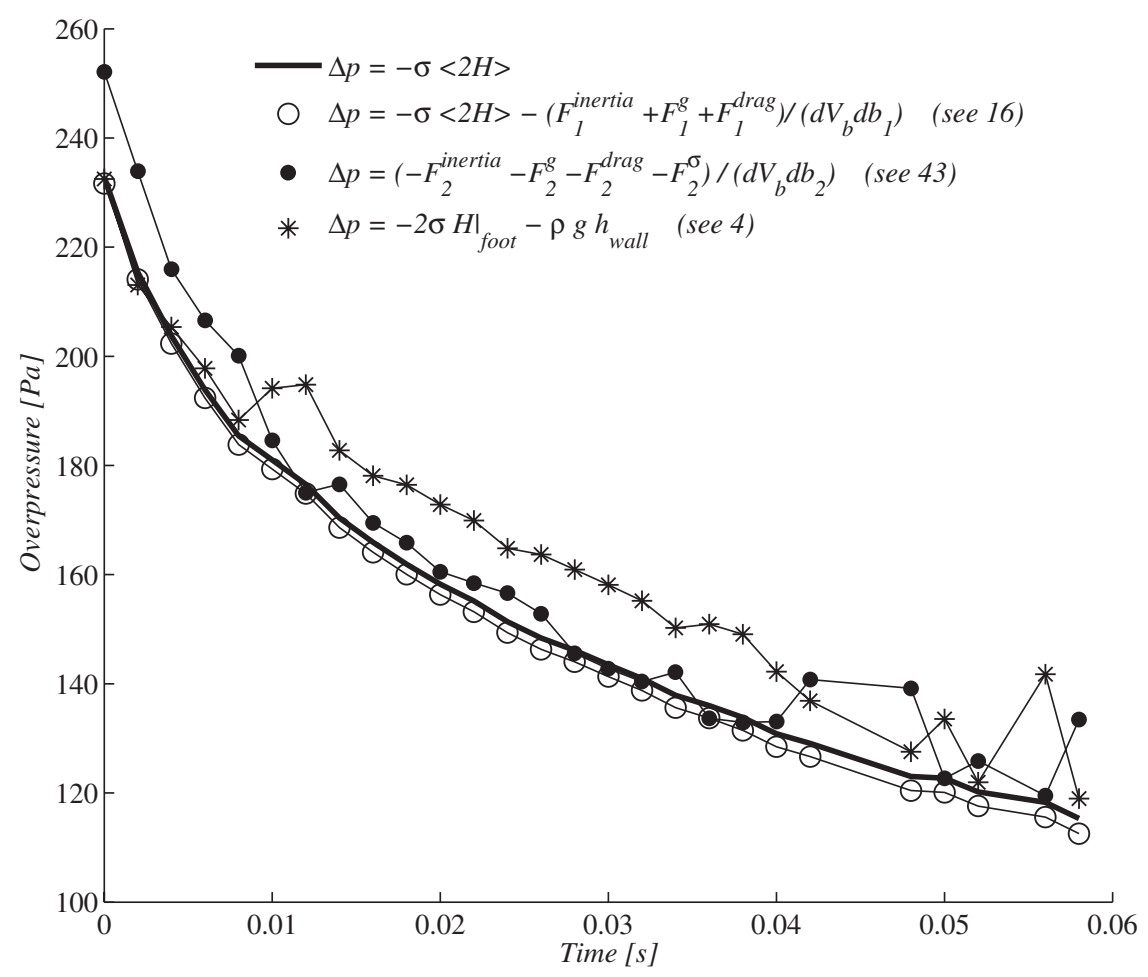

FIG. 10. Comparison of four computed histories of the pressure inside the bubble minus the hydrostatic pressure in the liquid at the wall for a terrestrial bubble.

previous solutions for this case of a wall chosen close to the actual wall. A fully independent way to determine the overpressure $\Delta p$ is given by the second Euler-Lagrange equation (17) which also comprises the overpressure as the only unknown. The $\Delta p$-values that make (17) satisfied identically, named $\Delta p_{2}$ in the following:

$$
\Delta p_{2}=-\left(F_{2}^{\sigma}+F_{2}^{\text {inertia }}+F_{2}^{g}+F_{2}^{d r a g}\right) /\left(\partial V_{b} / \partial b_{2}\right),
$$

are shown in Fig. 10 as well. The value of the relative difference $\left(\Delta p_{2}+\sigma\langle 2 H\rangle\right) /(-\sigma\langle 2 H\rangle)$ has for terrestrial bubbles been determined for 7 bubbles with typically 30 measured contours each. The average difference is found to be $4.3 \%$ with a standard deviation of $4 \%$.

The decrease in pressure inside the bubble with increasing time corresponds to a decrease in saturation temperature, $T_{\text {sat }}$, of the vapor in the bubble. This saturation temperature is equal to the temperature of the liquid at the interface, $T_{i n t}$. Since the pressure in the liquid is 1.1 bar with saturation temperature $37^{\circ} \mathrm{C}$, histories of $T_{\text {int }}$ can be computed from histories of $\Delta p$ as the one given in Fig. 10 with the aid of the Clausius-Clapeyron equation,

$$
\left(T_{i n t}-37^{\circ} \mathrm{C}\right)=\Delta p(273.15+37)\left(1 / \rho_{v}(273.15+37)-1 / \rho_{L}\right) / \Delta H .
$$

The molar mass of HFE7000 is $0.2 \mathrm{~kg} / \mathrm{mol}$ from which the mass density at $310.15^{\circ} \mathrm{C}, \rho_{v}(273.15$ +37 ), is computed with the ideal gas law. In the terrestrial case of Fig. $10, T_{\text {int }}$ is initially $37.06^{\circ} \mathrm{C}$ and decreases to $37.03{ }^{\circ} \mathrm{C}$. In the microgravity case, $T_{\text {int }}$ will be seen to be initially $37.13^{\circ} \mathrm{C}$ and decreasing to $37.05^{\circ} \mathrm{C}$. These values are consistent with the small wall superheating deduced from the growth histories above (about $1.2^{\circ} \mathrm{C}$ and $2.0^{\circ} \mathrm{C}$ ). These values also show that only a small decrease in saturation temperature, by $0.07^{\circ} \mathrm{C}$ in the microgravity case, accompanies the decrease in pressure inside the bubble.

The comparison of Fig. 10 is typical for all measurements and shows an agreement which is fair in view of the measurement accuracies. To the best of our knowledge this is the first time that the pressure inside a boiling bubble is determined from experiments in two independent ways simultaneously, with consistent values. Previous approximations in the literature at best invoked the 


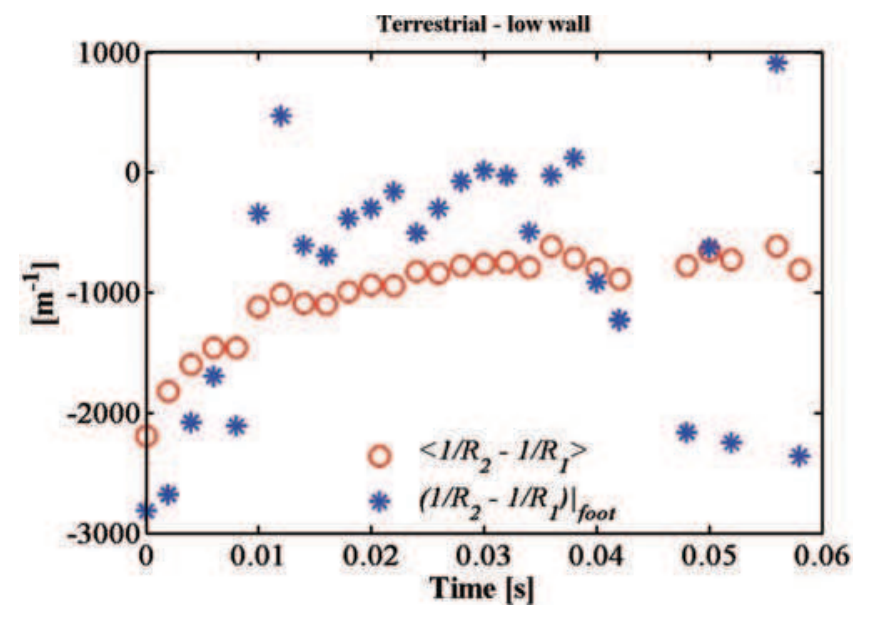

FIG. 11. Comparison of histories of curvature differences measured only at the foot (stars) with those measured at the entire liquid-gas interface (circles).

Laplace equation as a crude approximation to the governing equation (16). The above approximation $\Delta p=-\sigma\langle 2 H\rangle$ can be seen as a first improvement of the Laplace equation and a way to account for deformation of the bubble.

When two force contributions are measured and are nearly compensating, the relative error in their sum is usually large. The term $\left.\left(1 / R_{2}-1 / R_{1}\right)\right|_{\text {foot }}$ appears in the expression (18) which is an alternative for the sum of two major force contributions to the second Euler-Lagrange equation, the familiar force balance perpendicular to the wall in the direction of $h$. Figure 11 compares values of this term with the alternative $\left\langle 1 / R_{2}-1 / R_{1}\right\rangle$ which appears in (21). It is clearly seen that the scatter which occurs due to measurement difficulties near the foot in $\left.\left(1 / R_{2}-1 / R_{1}\right)\right|_{\text {foot }}$ is strongly reduced in $\left\langle 1 / R_{2}-1 / R_{1}\right\rangle$. The latter values are therefore expected to be more accurate and it will be shown in Sec. IV $C$ that this is indeed the case.

Histories of $1 /\left.R_{2}\right|_{\text {foot }}$ as determined from (23) have been compared with those of $-\sin \left(\theta_{c}\right) / r_{\text {foot }}$ and essentially the same values have been found. The change in the course of time is gradual for high-chosen walls and shows some scatter if the chosen wall is close to the actual wall. Once again the measurement accuracy in the proximity of the solid wall is found to be less. If values can alternatively be determined from interface-averaged quantities, such as $\langle 2 H\rangle$ and $\left\langle 1 / R_{2}-1 / R_{1}\right\rangle$, the method based on the entire interface is to be preferred. This is further demonstrated by the comparison of force components in $h$-direction below.

\section{Force balance normal to the wall for terrestrial bubbles}

In view of the accuracies found in Sec. IV B it stands to reason to consider the overpressure as determined from (16), or from $\Delta p=-\sigma\langle 2 H\rangle$, as the most accurate one. The resulting overpressure can be put into the $h$-equation (17), which also contains $\Delta p$ as the only unknown, yielding (22). However, the sum of the dominant forces, $F_{2}{ }^{\Delta p}+F_{2}{ }^{\sigma}$, is alternatively assessed with the aid of (18) or (21). As these two dominant forces are nearly compensating one another, the comparison of force components in $h$-direction of Fig. 12 only comprises the sum of these forces. In this figure, results for $F_{2}{ }^{\Delta p}+F_{2}{ }^{\sigma}$ of (18), (21) and (22) are compared with the solution of (17) with $\Delta p_{2}$ as defined in (43) of Sec. IV B: $F_{2}{ }^{\Delta p}+F_{2}{ }^{\sigma}=-F_{2}{ }^{\text {inertia }}-F_{2}{ }^{g}-F_{2}{ }^{\text {drag }}$.

Figure 12 shows that the contributions from drag and inertia are negligible for this terrestrial case. This is as expected and makes the fact that these two contributions are slightly overestimated irrelevant. Of the three ways to determine the sum $F_{2}{ }^{\Delta p}+F_{2}{ }^{\sigma}$, the one based on the difference in radii of curvature $\left\langle 1 / R_{2}-1 / R_{1}\right\rangle$, so on (21), is clearly closest to the values determined with $\Delta p_{2}$, in particular, at later times of the bubble growth process, i.e., for larger bubbles. Partly this is because of the increasing accuracy of measured parameters with increasing bubble growth. If the 


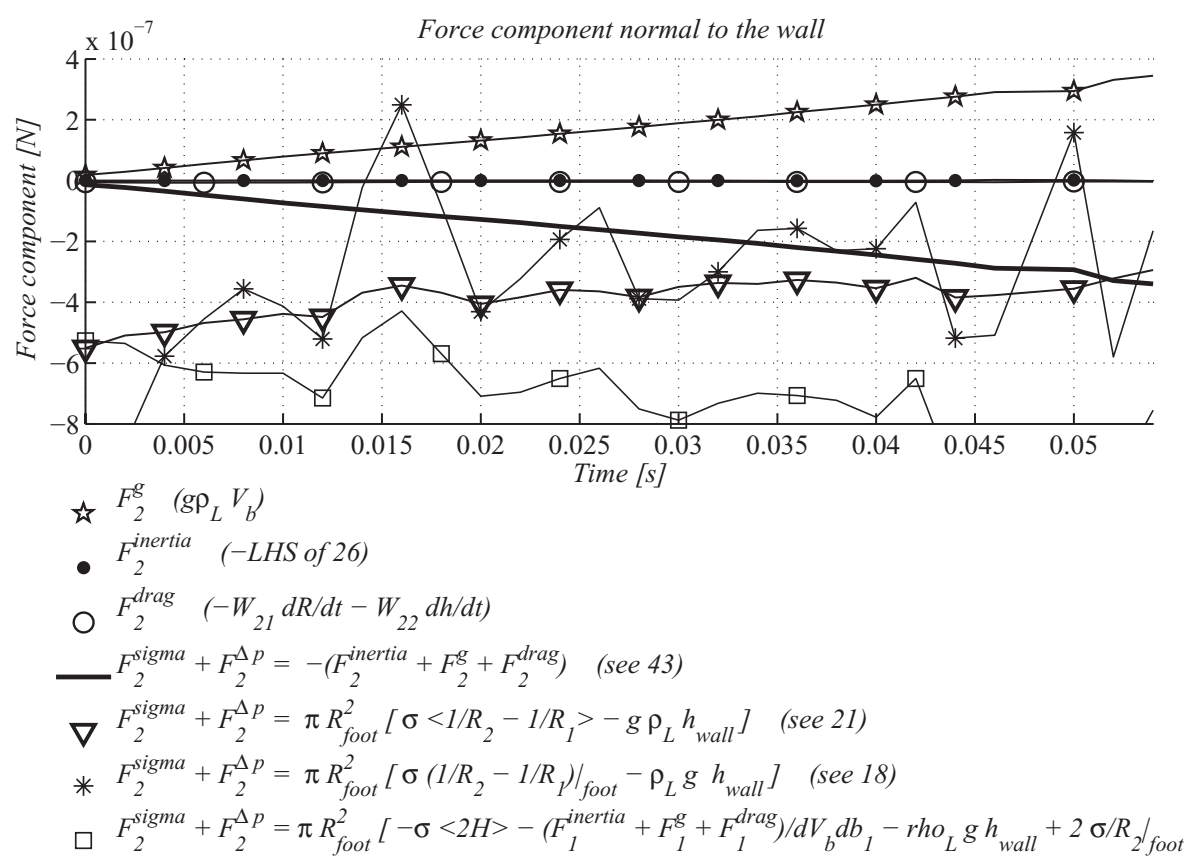

FIG. 12. Typical force component histories in the direction normal to the wall, anti-parallel to gravity, of a terrestrial bubble; the line indicated with open squares in the legend has been computed with (22). Note that if drag $F_{2}^{d r a g}=F_{22}^{\text {drag }}+F_{12}^{\text {drag }}$ $=-W_{21} \dot{R}-W_{22} \dot{h}$ would have been computed with equations of the type (39) the same result would have been obtained.

height of the chosen wall above the actual wall is increased, the averaging involved in (20) to obtain $\left\langle 1 / R_{2}-1 / R_{1}\right\rangle$ is performed over a reduced area. This usually reduces the accuracy of $\left(F_{2}{ }^{\Delta p}+F_{2}{ }^{\sigma}\right)$ a bit. For this reason, merely force balances with a chosen wall close to the actual wall are presented here.

\section{Force balances of microgravity bubbles}

All microgravity bubbles were found to detach the artificial cavity at an early stage during their growth and to move downstream close to or at the wall in the developing boundary layer there (Fig. 4). All microgravity bubbles were small as compared to terrestrial bubbles (Fig. 13). This happened due to different heating conditions of the bubble generator, although approaching velocity and temperature profiles of the liquid were kept the same. The area in the vicinity of the bubble foot is consequently difficult to be measured. The automated contour determination finds a bubble foot at the wall, i.e., $r_{\text {foot }} \neq 0$, but the mirage problem and/or other light refraction problems might be hiding an actual bubble shape, which is nearly spherical. At later stages of bubble growth, large bubbles are actually found to detach from the wall after being formed by coalescence of two smaller bubbles, see Fig. 4. If the bubble foot is actually zero, the sum of the two dominant forces in $h$-direction, $F_{2}{ }^{\Delta p}+F_{2}{ }^{\sigma}$, must be zero. In addition, both the gravity constant and the volume are small which makes that the main force which is compensating the body force, $F_{2}{ }^{\Delta p}+F_{2}{ }^{\sigma}$, must also be small. Small values of this sum are therefore anticipated, as is further discussed below.

Gravity in the form of $g$-jitter is found to have values of the same order of magnitude than the inertia and drag estimates, both in the $b_{1}$ and $h$-equation. Note that the $g$-jitter values were actually measured and used in the analysis via a time-dependent $g$-value. The $g$-jitter was measured to have a mean value of zero and maximum values of about $\pm 0.5 \mathrm{~m} / \mathrm{s}^{2}$. Inertia and drag are counteracting and both are small in the governing $b_{1}$-equation, and consequently the overpressure as determined by $-\sigma\langle 2 H\rangle$ is nearly the same as the overpressure $\Delta p_{2}$ determined from the full governing equation (16), see Fig. 14. 


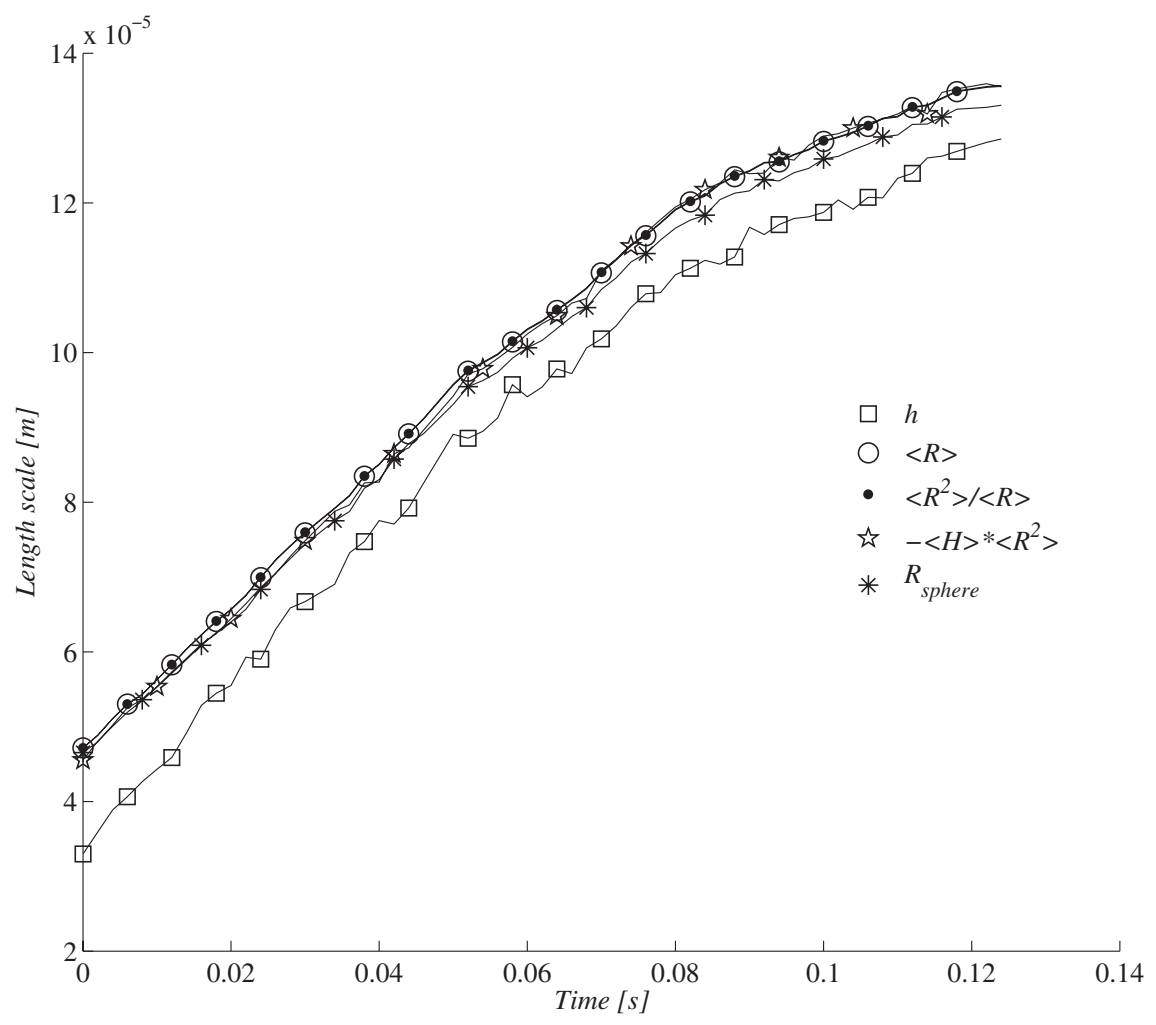

FIG. 13. Comparison of length scale histories for a microgravity bubble. The bubble does not appear to take off, since $R_{\text {sphere }}$ exceeds $\mathrm{h}$ at all times, although it slides downstream along the wall.

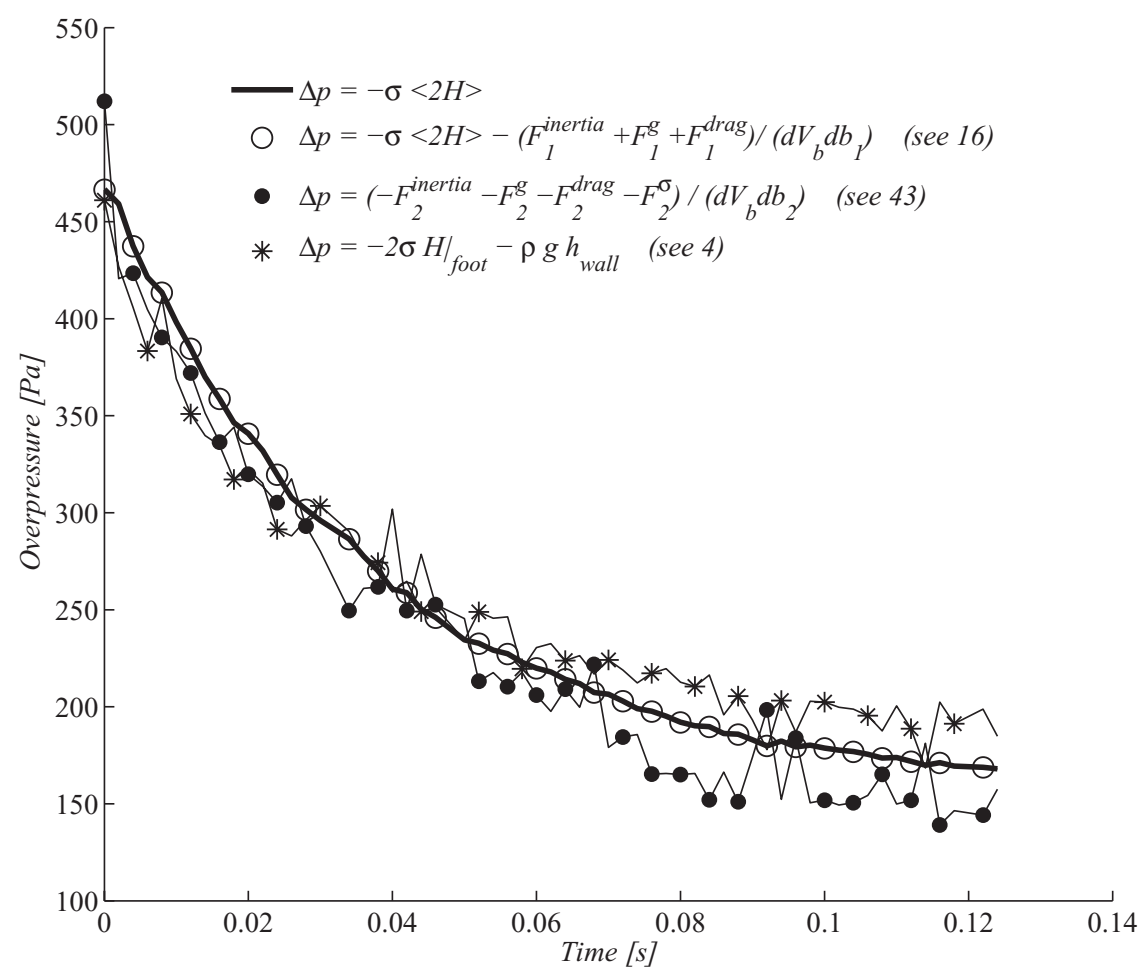

FIG. 14. Comparison of four computed histories of the pressure inside the bubble minus the hydrostatic pressure in the liquid at the wall for a microgravity bubble. 


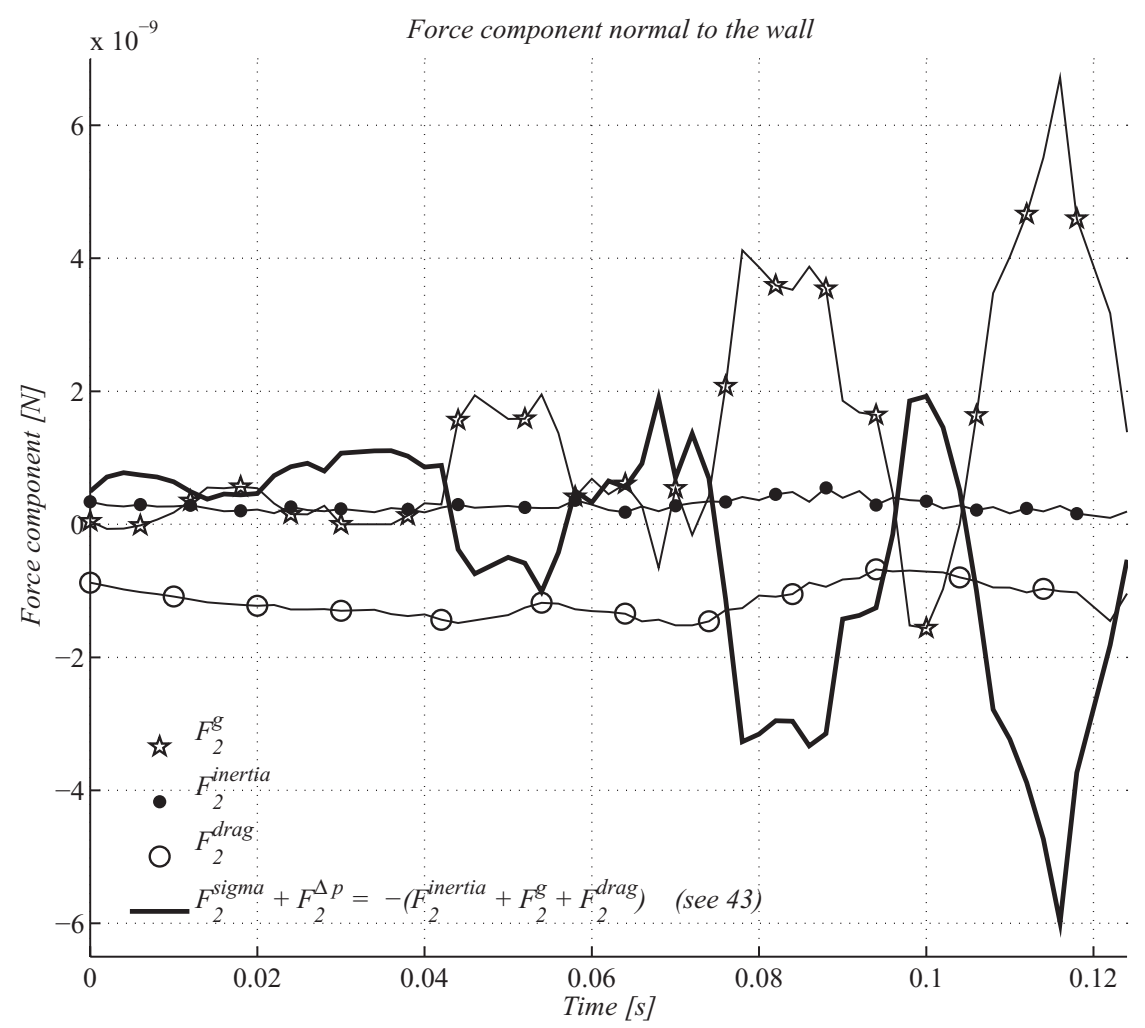

FIG. 15. Typical histories of force component normal to the wall in microgravity showing the significance of drag; the same symbols as in the terrestrial case of Fig. 12 are used. The acceleration $g$ of the gravity component $\left(g \rho_{L} V_{b}\right)$ is measured with acceleration meters, inertia is computed for a truncated sphere shape (minus the LHS of (26)) and drag is slightly overestimated in the Levich approach for the truncated sphere shape by $F_{2}^{d r a g}=-W_{21} \dot{R}-W_{22} \dot{h}$. The sum $F_{2}{ }^{\Delta p}+F_{2}{ }^{\sigma}$ is only shown for the $\Delta p_{2}$-case of (43); the other experimental assessments of this sum (see legend of Fig. 12) are fluctuating around zero but are too inaccurate to be shown.

The relative difference $\left(\Delta p_{2}+\sigma\langle 2 H\rangle\right) /(-\sigma\langle 2 H\rangle)$ has been determined for 6 microgravity bubbles, each with about 50 measured contours, with an average result of $-6.0 \%$ with a standard deviation of $9 \%$. The scatter in the overpressure determination is in the case of microgravity obviously higher than in the case of terrestrial bubbles due to a higher measurement inaccuracy because of the smaller size of the bubbles.

In the force balance normal to the wall (Fig. 15), inertia is estimated to be mainly due to lift related to the approaching velocity since bubble growth is slow and other inertia terms consequently small. Note that the estimates of inertia contributions are fairly accurate since the actually measured shapes are close to those of truncated spheres. Due to the small size of the bubbles, the measuring accuracy of $F_{2}{ }^{\Delta p}+F_{2}{ }^{\sigma}$ with (21) or with other expressions is not high. Moreover, in (18) or similar expressions for the $h$-equation, for example, the one employing (22), the estimates of drag and inertia appear to be counteracting although drag is not particularly small at early times (Fig. 15). As a result, $F_{2}{ }^{\Delta p}+F_{2}{ }^{\sigma}$ is nearly compensating $g$-jitter at all times and hence fluctuating around zero. Lift on microgravity bubbles is smaller than $g$-jitter and nearly negligible as anticipated. However, at early times of bubble growth, when the drag error estimates are less than $20 \%$, our drag computations turn out to be exceeding both $g$-jitter and the sum $\left(F_{2}{ }^{\Delta p}+F_{2}{ }^{\sigma}\right)$. Even allowing for a $40 \%$ error at later times of bubble growth, drag is contributing then and both drag and $g$-jitter induced buoyancy are found to be important during bubble growth in microgravity. This might seem surprising at first sight, but it merely shows how small the sum $\left(F_{2}{ }^{\Delta p}+F_{2}{ }^{\sigma}\right)$ is and how close the bubble shape is to that of a truncated sphere. The importance of drag normal to the wall implies that drag also parallel to the wall might become important, see Sec. III E. The sliding away of the bubbles, despite the 
possibility of staying pinned at the cavity mouth as measured in the on-ground experiments, is a clear indication of this.

Bigger bubbles with a clear footing at the wall are required to make the force assessment of the $h$-force component $F_{2}{ }^{\Delta p}+F_{2}{ }^{\sigma}$ accurate. Only with measurements of such bigger bubbles a meaningful comparison can be made of measured and predicted values of the sum $\left(F^{\text {inertia }}+F^{\text {drag }}\right.$ $\left.+F^{g}\right)$, with the gravity force $F^{g}$ based on measured $g$-jitter histories. Within the present measurement accuracy, bubbles could well be detached from the wall already during their growth, while sliding downstream along the bubble generator. The authors believe this to be the case as capillary forces with such high curvatures are high and motion of a dry patch at the wall is difficult to imagine at the low liquid velocity employed. Interpretation of measurements of sliding bubbles in the literature ${ }^{25}$ is hampered by the same observation difficulties.

In case a bubble is found to jump away from the wall, momentarily after coalescence (Fig. 4), it will immediately go back to the wall. As there is no preferred direction in parabolic flights related to gravity, the direction of the main flow and the geometry must account for the systematic preference of bubbles to stick to the wall. Since bubble growth is slow and bubbles exist for quite some time in the boundary layer, sufficient time is available for bubbles to collect vorticity in their wake. The reason why bubbles that grow and move downstream in the boundary layer stay close to the wall of the bubble generator is therefore believed to be a pressure gradient due to inhomogeneous vorticity distributions, although there is no direct evidence for this.

\section{E. Absence of inert gases proven from the force balances}

An interesting feature of the equations presented in Sec. III is the possibility to accommodate for variations in surface tension coefficient due to variations in interfacial temperature. Such a variation might occur in case degassing would not have been performed properly and gas remnants would occur in the content of the gas/vapor bubble. Variation in partial pressure along the interface would then cause variation in interfacial saturation temperature and Marangoni flows would occur in the liquid. Instead of $\sigma\langle 2 H\rangle$, the average $\langle\sigma 2 H\rangle$ is used in the first Euler-Lagrange equation now. Equation (10) is adapted in a similar way to yield another version of the second Euler-Lagrange equation. This procedure seems to be a natural way to take the force due to Marangoni convection into account.

We investigated the consequences of possible gas remnants by prescribing a linear variation of temperature with $x$ along the interface, which is a linear variation with height in the case of a truncated sphere. Surface tension coefficient was assumed to vary linearly with temperature from $11.4 \mathrm{mN} / \mathrm{m}$ at $34{ }^{\circ} \mathrm{C}$ to $9.36 \mathrm{mN} / \mathrm{m}$ at $56^{\circ} \mathrm{C}$. The capillary term (10) turned out to be more sensitive to variations in surface tension than the term $\langle\sigma 2 H\rangle$ of the other Euler-Lagrange equation. Only a moderate variation in surface tension coefficient, from 0.0119 to $0.0108 \mathrm{mN} / \mathrm{m}$ over the height in the last stage of growth of a (large) terrestrial bubble, sufficed to give a notable difference in overpressure $\Delta p$ of about $5 \%$ at all times. However, with the highest temperature at the wall the overpressure was increased as compared to overpressure values for a homogeneous surface tension coefficient. A not realistic negative gradient, with a lowest temperature at the wall, would be required to annihilate remaining differences between $\Delta p_{2}$ and $-\sigma\langle 2 H\rangle$ for terrestrial bubbles. It is concluded that degassing was done properly and that pure vapor bubbles have been measured during the terrestrial measurements. The scatter in the values of the difference $\left(\Delta p_{2}+\sigma\langle 2 H\rangle\right)$ is high for microgravity bubbles and is not reduced by invoking a realistic surface tension gradient along the interface. As the degassing procedure was the same for parabolic flights as on earth it is concluded that pure vapor bubbles have been measured in all measurement conditions.

\section{F. Force balance parallel to the wall in microgravity}

In principle, the motion of a boiling bubble parallel to the wall after detachment from the artificial site can be described by a force balance parallel to the wall. The obvious additional generalized coordinate is the distance of the center to the frontal edge of the bubble generator. In order to estimate the drag force in this direction, the shape asymmetry between upstream and downstream halves of 
the bubble needs to be taken into account as well as boundary layer development and interaction between neighboring bubbles. The last interaction is even in fully developed channel flow a laborious task. The measurements in microgravity reported in the present paper have revealed a sometimes erratic motion of bubbles parallel to the wall, partly connected to $g$-jitter. Even motion upstream has been observed. The sum of the two counteracting overpressure and capillary forces, $F^{\Delta p}+F^{\sigma}$, may accommodate also in flow direction for $g$-jitter and other effects via minor bubble deformations. There is no method available in the literature to estimate the drag force parallel to the wall for all these bubble shapes. Computations of this drag force require knowledge of the flow field and in particular the vorticity field which is strongly affected by the erratic motion due to $g$-jitter and the presence of wakes downstream of neighboring bubbles. The computation of inertia forces must take full account of the asymmetry of the bubble shape in flow direction, and is therefore essentially 3D. Lastly, the experimental assessment of $F^{\Delta p}+F^{\sigma}$ requires a high spatial resolution of the recorded images. For all these reasons, no attempt is made to assess the force balance parallel to the wall here.

\section{CONCLUSIONS}

Terrestrial and microgravity experiments were carried out with the same test rig, comprising a locally heated artificial cavity in the center of a channel near the frontal edge of an intrusive glass bubble generator. The approaching liquid flow velocity is laminar $(1.2 \mathrm{~cm} / \mathrm{s})$ and parabolic and a boundary layer develops downstream from the frontal edge of the bubble generator. Bubbles in microgravity were found to leave the cavity and move downstream in the boundary layer developing at the bubble generator; the terrestrial bubbles grew only at the cavity. Bubble shapes were generally not far from those of truncated spheres, which provoked the usual wish to set up an analysis of forces on the bubble in a way that comparison with the analysis of a truncated sphere is facilitated. This necessitated the definition of a "sphere radius," $R_{\text {sphere }}$, and required careful handling of fit parameter $\tilde{b}_{2}$. The reward was the possibility to limit the analysis to only two governing equations whereas more complex analysis involves ${ }^{8}$ more governing equations.

A generalization of the Laplace equation is found: $\Delta p=-\sigma\langle 2 H\rangle$, which applies to a deforming bubble attached to a plane wall. Here, $\Delta p$ is the difference in static pressures inside the bubble and in the liquid close to the wall, $2 H$ denotes local mean curvature and \langle\rangle averaging over the gasliquid interface. This generalization stems from the first Euler-Lagrange equation for the isotropic component of the radial distance of the bubble contour to the center of the bubble. A fully independent way to determine the overpressure $\Delta p$ is given by the second Euler-Lagrange equation for the distance $h$ of the center of the bubble to the wall, Eq. (17). The $\Delta p$-values that make (17) satisfied identically have been named $\Delta p_{2}$ and relative difference $\left(\Delta p_{2}+\sigma\langle 2 H\rangle\right) /(-\sigma\langle 2 H\rangle)$ has for terrestrial bubbles measured been found to be $4 \%$. For microgravity bubbles measured this difference was about $6 \%$. To the best of our knowledge this is the first time that the pressure inside a boiling bubble is determined from experiments in two independent ways simultaneously, with values in agreement to within $5 \%$.

A way is found to determine the sum of the two counteracting major force contributions on a bubble in the direction normal to the wall, overpressure force $F_{2}{ }^{\Delta p}$ and capillary force $F_{2}{ }^{\sigma}$, from a single, directly measurable quantity, $\left.\sigma\left(1 / R_{2}-1 / R_{1}\right)\right|_{\text {foot }}$. This is the difference of the two radii of curvature at the bubble foot. The value of the difference in radii of curvature averaged over the liquid-vapor interface is $\left\langle\sigma\left(1 / R_{2}-1 / R_{1}\right)\right\rangle$. Measurement accuracy obtained with the aid of $\left\langle\sigma\left(1 / R_{2}-1 / R_{1}\right)\right\rangle$ is far better than that of other methods. For larger terrestrial boiling bubbles the resulting measurement accuracy if found to be sufficient to find values for $\left(F_{2}{ }^{\Delta p}+F_{2}{ }^{\sigma}\right)$, which are neatly compensating the buoyancy force, i.e., which are in agreement with $\left(F_{2}{ }^{\Delta p}+F_{2}{ }^{\sigma}\right)$-values as determined with the aid of $\Delta p_{2}$.

The new analysis methods of the main force components (overpressure, capillary, body forces) permit the accounting for a surface tension gradient along the liquid-vapor interface. As realistic gradients would increase the difference between the overpressures determined in the above-mentioned two independent ways it is concluded that no such gradients occurred. This confirms the thoroughness of the degassing procedure. 
To the best of our knowledge, the expressions presented for lift and drag are the most appropriate ones for the terrestrial and microgravity bubbles measured in our experiments. However, other expressions for $F^{v o r t}$ and $F^{\text {drag }}$ could easily be combined with the generally valid expressions derived for $F^{\Delta p}, F^{\sigma}$, and $F^{g}$, following Eq. (2). In addition, more complex deformation is easily accommodated by taking more generalized coordinates into account in the assessment of $F_{2}$ inertia $\left(b_{j}, \dot{b}_{j}, \ddot{b}_{j}\right)$ and $F_{2}{ }^{d r a g}\left(b_{j}, \dot{b}_{j}\right)$. The other forces already take account of these full dependencies in the present study. The expressions for drag and lift are recommended for growing boiling bubbles if either $R e_{1} \gg 1$ or $R e_{2} \gg 1$, or if both $R e_{1} \geq 1$ and $R e_{2} \geq 1$ and if either $R e_{V} \gg 1$ or the lifetime of bubbles is short.

Bubble shapes of microgravity bubbles were generally close to those of truncated spheres. For these bubbles, lift is smaller than $g$-jitter, and therefore nearly negligible as anticipated. However, at early times of bubble growth drag our drag computations turn out to be exceeding both $g$-jitter and the sum $\left(F_{2}{ }^{\Delta p}+F_{2}{ }^{\sigma}\right)$, while the drag error estimates are less than $20 \%$. Even allowing for a $40 \%$ error at later times of bubble growth, the findings show that both drag and $g$-jitter are important during bubble growth in microgravity. On earth, gravity is dominant, bubble deformation is somewhat stronger and both lift and drag are negligible as expected.

Summarizing, the main results of the paper are:

1. A rigorous method to assess capillary, overpressure, and body forces during bubble growth from recorded images taking full account of arbitrary deformation. The method followed to assess inertial lift and viscous potential drag can be extended to arbitrarily deforming bubbles but is here limited to the two main components, $h$ and $b_{1}$. This is appropriate in view of the measured bubble shapes being close to those of truncated spheres.

2. A generalization of the Laplace equation to connect the pressures in the bubble and at its foot for deformed bubbles at a wall.

3. A new solution for the problem of determining the sum of the two dominant force components while in convective boiling the bubble foot is difficult to be observed and one of the these two forces is critically dependent on this bubble foot. These two force components are counteracting such that the sum is several orders of magnitude smaller than each individual component. The sensitivity to the bubble shape is such that the bubble easily accommodates to external disturbances by tiny adaptations of its shape.

4. A determination, from new experiments and with the above analyzing method, of histories of bubble pressure in two independent ways, with values in agreement to within $5 \%$.

5. The findings that both drag and the body force due to $g$-jitter are important in parabolic flights.

6. A way to determine whether surface tension gradients occur during bubble growth in experiments or not.

7. The experimental finding that in identical flow conditions different superheats required for incipience of boiling at an artificial nucleation site in microgravity and on ground. In microgravity, bubbles start sliding but are probably fully detached, whereas in terrestrial experiments bubbles directly lift off from the artificial nucleation site. Boundary layer development is affected by mixed convection in on-ground measurements, but merely by forced convection in microgravity.

\section{ACKNOWLEDGMENTS}

Measurements taken in parabolic flight have been supported by Centre National d'Études Spatiales, by the European Space Agency and Novespace. The contribution of V. H. Pereira da Rosa was made possible by financial support of the European Community within the framework of the European Brazilian Windows program. Boiling research at Eindhoven University of Technology is currently supported by the Dutch Technology Foundation denoted by STW (Stichting Technische Wetenschappen), applied science division of Nederlandse Organisatie voor Wetenschappelijk Onderzoek and the Technology program of the Ministry of Economic Affairs. 


\section{APPENDIX: ADDED MASS AND DRAG COEFFICIENTS}

The following fits of computed data are on a finite domain in order to have a high-accuracy fit for each coefficient, with $r^{2}$-values of typically 0.9995 and $F$-values of typically 75000 . By definition, $W_{i j}=\pi \mu b_{1} \hat{W}_{i j}$ and $\lambda=b_{1} /(2 h)$; the value of $\lambda$ goes to infinity if the truncated sphere approaches that of a hemisphere.

$$
\begin{aligned}
\text { If } \lambda \geq & 0.5 \text { and } \lambda<0.52, \text { then } \\
\hat{W}_{11}= & -5380203.17695 \lambda^{3}+8300488.103047 \lambda^{2}-4268331.30233 \lambda+731589.643606, \\
\hat{W}_{12}= & 4055714.74201 \lambda^{3}-6259115.82719 \lambda^{2}+3219715.931029 \lambda-552049.750722, \\
\hat{W}_{22}= & -2720491.72838 \lambda^{3}+4200956.306963 \lambda^{2}-2162358.20635 \lambda+371016.142295 . \\
\text { If } \lambda \geq & 0.52 \text { and } \lambda \leq 1, \text { then } \\
\hat{W}_{11}= & \left(-93059.475156+710538.111722 \lambda-2246394.17527 \lambda^{2}+3773911.729744 \lambda^{3}\right. \\
& \left.-3553791.26722 \lambda^{4}+1778050.692458 \lambda^{5}-369154.914859 \lambda^{6}\right)^{0.5}, \\
\hat{W}_{12}= & \exp \left(184.303261-1178.375359 \lambda+3130.822666 \lambda^{2}-4438.214264 \lambda^{3}+3550.461665\right. \\
& \left.\lambda^{4}-1521.598072 \lambda^{5}+273.123658 \lambda^{6}\right), \\
\hat{W}_{22}= & 1 /\left(-32.531609+257.429857 \lambda-839.643949 \lambda^{2}+1452.543662 \lambda^{3}-1404.648165 \lambda^{4}\right. \\
& \left.+719.734252 \lambda^{5}-152.651839 \lambda^{6}\right) . \\
\text { If } \lambda \geq & 0.5 \text { and } \lambda \leq 1, \text { then } \\
\alpha= & 111.62137-844.131315 \lambda+2678.058461 \lambda^{2}-4534.349913 \lambda^{3}+4311.889654 \lambda^{4} \\
& -2180.345705 \lambda^{5}+457.591961 \lambda^{6}, \\
\psi= & 220.824854+1639.114567 \lambda-5130.691427 \lambda^{2}+8625.857798 \lambda^{3}-8169.91248 \lambda^{4} \\
& +4121.492877 \lambda^{5}-863.784836 \lambda^{6}, \\
v= & 104.601303-736.214699 \lambda+2293.784611 \lambda^{2}-3857.878559 \lambda^{3}+3659.955261 \lambda^{4} \\
& -1849.854303 \lambda^{5}+388.412909 \lambda^{6}, \\
\alpha= & 0.359528+1.341274 \lambda-1.973813 \lambda^{2}+0.796613 \lambda^{3} .
\end{aligned}
$$

A matlab file to compute these functions is available on request.

${ }^{1}$ S. van Stralen and R. Cole, Boiling Phenomena, Vol. 1 and 2 (McGraw Hill, New York, 1979).

${ }^{2}$ J. P. Kroes, C. W. M. van der Geld, and E. van Velthooven, "Evaluation of four nucleate flow boiling models," in Advances in Multiphase Flow and Heat Transfer (Bentham Science Publishers, Sharjah, 2009), Vol. 1, pp. 267-283.

${ }^{3}$ N. Basu, G. Warrier, and V. Dhir, "Wall heat flux partitioning during subcooled flow boiling: Part 1—Model development," J. Heat Transfer 127, 131-140 (2005).

${ }^{4}$ C. W. M. van der Geld, "The dynamics of a boiling bubble before and after detachment," Heat Mass Transfer 45, 831-846 (2009).

${ }^{5}$ A. K. Chesters, "An analytical solution for the profile and volume of a small drop or bubble symmetrical about a vertical axis," J. Fluid Mech. 81(4), 609-624 (1977).

${ }^{6}$ G. Son, V. K. Dhir, and N. Ramanujapu, "Dynamics and heat transfer associated with a single bubble during nucleate boiling on a horizontal surface," J. Heat Transfer 121, 623-631 (1999).

${ }^{7}$ C. W. M. van der Geld, "On the motion of a spherical bubble deforming near a plane wall," J. Eng. Math. 42(2), 91-118 (2002).

${ }^{8}$ C. W. M. van der Geld and J. G. M. Kuerten, “Axisymmetric dynamics of a bubble near a plane wall,” J. Fluid Mech. 640, 267-305 (2009).

${ }^{9}$ C. W. M. van der Geld, "Shape oscillations of a boiling bubble," Multiphase Sci. Technol. 22(2), 157-175 (2010).

${ }^{10} \mathrm{G}$. Duhar and C. Colin, "A predictive model for the detachment of bubbles injected in a viscous shear flow with small inertial effects," Phys. Fluids 16(5), L31 (2004).

${ }^{11}$ G. Duhar and C. Colin, "Dynamics of bubble growth and detachment in a viscous shear flow," Phys. Fluids 18, 077101 (2006).

12 J. F. Klausner, R. Mei, D. M. Bernhard, and L. Z. Zeng, "Vapor bubble departure in forced convection boiling," Int. J. Heat Mass Transfer 36, 651-662 (1993).

${ }^{13}$ L. Z. Zeng, J. F. Klausner, D. M. Bernhard, and R. Mei, "A unified model for the prediction of bubble detachment diameters in boiling systems-II. Flow boiling,” Int. J. Heat Mass Transfer 36, 2271-2279 (1993).

${ }^{14}$ P. G. Saffman, Vortex Dynamics (Cambridge University Press, Cambridge, 1992), p. 2.

${ }^{15}$ J. Magnaudet and I. Eames, "The motion of high-Reynolds-number bubbles in Inhomogeneous flows," Annu. Rev. Fluid Mech. 32, 659-708 (2000).

${ }^{16}$ D. B. R. Kenning, "Optical studies of boiling heat transfer: insights and limitations," Int. J. Heat Fluid Flow 25, 209-222 (2004).

17 J. C. Slattery, Interfacial Transport Phenomena (Springer-Verlag, New York, 1990). 
${ }^{18}$ V. G. Levich, Physicochemical Hydrodynamics (Prentice Hall, Englewood Cliffs, 1962).

${ }^{19}$ D. Legendre, C. Colin, and T. Coquard, "Lift, drag and added mass of a hemispherical bubble sliding and growing on a wall in a viscous linear shear flow," Philos. Trans. R. Soc. London, Ser. A 366, 2233-2248 (2008).

${ }^{20}$ J. Magnaudet and D. Legendre, "The viscous drag force on a spherical bubble with a time-dependent radius," Phys. Fluids 10(3), 550 (1998).

${ }^{21}$ G. K. Batchelor, An Introduction to Fluid Dynamics (Cambridge University Press, Cambridge, 1967).

${ }^{22}$ P. M. Lovalenti and J. F. Brady, "The hydrodynamic force on a rigid particle undergoing arbitrary time-dependent motion at small Reynolds number,” J. Fluid Mech. 256, 561-605 (1993).

${ }^{23}$ H. Schlichting and K. Gersten, Boundary Layer Theory, 8th ed. (Springer-Verlag, New York, 2000).

${ }^{24}$ G. Duhar, G. Riboux, and C. Colin, "Vapour bubble growth and detachment at the wall of shear flow," Heat Mass Transfer 45, 847-855 (2009).

${ }^{25}$ R. Situ, T. Hibiki, M. Ishii, and M. Mori, "Bubble lift-off size in forced convective subcooled boiling flow," Int. J. Heat Mass Transfer 48, 5536-5548 (2005). 